\title{
A comparative study of sound generation by laminar, combusting and non-combusting jet flows
}

\author{
Mohsen Talei • Michael J. Brear • \\ Evatt R. Hawkes
}

Received: date / Accepted: date

\begin{abstract}
Sound production by two-dimensional (2D), laminar jet flows with and without combustion is studied numerically and theoretically. The compressible Navier-Stokes, energy and progress variable equations are solved by resolving both the near field and the acoustics. The combusting jet flows are compared to non-combusting jets of the same jet Mach number, with the non-combusting, non-isothermal jets having the same steady temperature difference as the combusting jets. This infers that the magnitude of entropic and density disturbances is similar in some of the combusting and non-combusting cases. The flows are perturbed by a sinusoidal inlet velocity fluctuation at different Strouhal numbers. The computational domain is resolved to the far-field in all cases, allowing direct examination of the sound radiated and its sources.

Lighthill's acoustic analogy is then solved numerically using Green's functions. The radiated sound calculated using Lighthill's equation is in good agreement with that from the simulations for all cases, validating the numerical solution of Lighthill's equation. The contribution of the source terms in Dowling's reformulation of Lighthill's equation is then investigated. It is shown that the source term relating to changes in the momentum of density inhomogeneities is the

M. Talei

Department of Mechanical Engineering,

University of Melbourne, VIC 3010, Australia

Tel.: +61-3 83446318

Fax: +61-3 83444290

E-mail: mohsen.talei@unimelb.edu.au

M. J. Brear

Department of Mechanical Engineering,

University of Melbourne, VIC 3010, Australia

Tel.: +61-3 83446722

Fax: +61-3 83444290

E-mail: mjbrear@unimelb.edu.au

E. R. Hawkes

School of Mechanical and Manufacturing Engineering/School of Photovoltaic and Renewable Energy Engineering,

University of New South Wales, Sydney, NSW 2052, Australia

Tel.: +61-2 93856155

Fax: +61-2 93857762

E-mail: evatt.hawkes@unsw.edu.au
\end{abstract}


dominant source term for all non-reacting, non-isothermal cases. Further, this source term has similar magnitude in the combusting cases, and is one of several source terms that have similar magnitude to the source term involving fluctuations in the heat-release rate.

Keywords premixed · combustion - flame direct numerical simulation · sound generation · combustion instability $\cdot$ annihilation

\section{Introduction}

Sound generation by combusting jet flows has been the subject of considerable research, particularly over the last sixty years. Reducing noise from devices such as aircraft engines, industrial burners and diesel engines has motivated many researchers to study noise generation by different types of combusting flows [e.g. 50, 57]. Combustion-generated sound has additional importance since its interaction with the flame may lead to thermo-acoustic instability, for example in rockets or gas-turbines [e.g. 7, 19, 35, 36, 49]. To reduce the noise in such devices, the mechanisms of sound generation by reacting jet flows should be understood.

So-called 'acoustic analogies' can be used to obtain the radiated sound by jet flows and investigate the sound-generation mechanisms. Acoustic analogies are a rearrangement of the equations of fluid motion into various inhomogeneous wave equations. Lighthill [37] proposed the first and best known acoustic analogy by rearranging the continuity and momentum equations only. Since then, Lighthill's equation has been used widely to investigate the mechanism of sound generation by different jet flows $[7,11]$.

Several other acoustic analogies have also been developed. For example, Phillips' and Lilley's acoustic analogies account for the interaction with the mean flow using substantial derivatives for the wave operator [38, 44]. Goldstein [23] derived a linearised form of Lilley's equation to decompose the unsteadiness of the flow to non-acoustic and acoustic parts, introducing first and second order solutions. Goldstein [24] then introduced a more general acoustic analogy which presented a new approach for defining the base flow.

In the case of combusting flows, Strahle's work [51] appears to be the first to use an acoustic analogy to estimate the radiated sound. Strahle [51] argued that part of Lighthill's stress tensor featuring the density fluctuations is the dominant source term of Lighthill's equation. He then presented two estimates for the acoustic power based on wrinkled flame and distributed reaction models. In his subsequent work [52], he reformulated this monopolar source term as a function of heat-release rate fluctuations and argued that the far-field sound can be estimated regardless of the turbulence structure and flame type. This result is consistent with the experimental and theoretical study by Hurle et al. [28] and other earlier works, but provided a more fundamental explanation as to why sound generation by flames is commonly monopolar. Indeed, variations in the heat-release rate are now commonly considered to have a significant effect on sound generation [e.g. 9, 10, 18, 25, 26, 27, 36, 47, 53, 54, 55].

Direct Numerical Simulation (DNS) [e.g. 22, 31, 40, 60] and Large Eddy Simulation (LES) [e.g. 2, 5, 29, 30] have also been used to study sound generation by reacting and non-reacting flows. LES has perhaps received more attention since it is computationally less expensive and is also suitable for higher Reynolds numbers. On the other hand, since all relevant features of the flame and flow are directly resolved and source terms can be directly calculated, DNS enables more detailed investigation. DNS of sound generation from a turbulent premixed flame has not yet been reported and is likely to be computationally expensive. However, the simpler situation of a forced laminar flame still contains relevant dynamics of flow-flame interactions and their effects on sound generation. This approach has been used in several experimental studies [e.g. 8, 33, 47, 48]. 
One focus of the present paper is therefore to simulate several combusting and noncombusting, laminar jet flows by resolving both the near field and the far field. Simple chemistry is a logical first step is used for the combusting cases, and detailed chemistry studies are needed for the future. The solution of Lighthill's acoustic analogy on these cases will be validated, and then used to examine the different source terms. Indeed, this appears to be the first time that this equation has been closed on a combusting jet flow, providing a solid basis on which to discuss the relative magnitude of different source terms in Lighthill's equation.

This approach is analogous to the non-combusting study of Colonius et al. [12], and differs from the approach taken in two other recent works by the authors $[4,56]$. In one of these studies [4], extended version of Myers' disturbance energy corollary [43] was closed on a confined laminar flame, and thus examined the utility of the famous Rayleigh criterion. In another work [56], the authors examined the validity of flamelet modelling by considering only the heat release source term in Dowling's reformulation of Lighthill's equation for three cases of different Lewis numbers. However, in the present study, Lighthill's equation is closed on a number of combusting and non-combusting laminar jets by developing a solution method that considers the contribution of boundary conditions such as inflow excitation, reflections from the inflow and unclosed source regions. Also, the complete set of source terms in Dowling's reformulation are considered to investigate the contribution of each source term for all cases studied.

The present study has two parts. First, acoustically excited premixed flames and nonreacting jet simulations that resolve both the jet flow and the acoustics will be examined. A numerical solution of Lighthill's equation then enables comparison of the source terms of Dowling's reformulation of Lighthill's equation [18] over a range of forcing frequencies.

The following test cases are considered (see Figure 1 and Table 1).

Case NR1: A non-reacting isothermal flow, in which the temperature of the jet is the same as the far-field temperature.

Case NR2: A non-reacting 'cold' flow, in which a jet at the same temperature as Case 1, enters a domain with the same temperature as the burnt gas in Cases 4 and 5 below.

Case NR3: A non-reacting 'hot' flow, in which a jet with the same temperature as the burnt gas in Cases 4 and 5 below, enters a domain with the same far-field temperature as Case 1.

Cases R4a-e: Premixed laminar flames, surrounded by a far-field that is uniformly at the flame's adiabatic temperature. Five simulations with different inlet Mach numbers, Reynolds numbers, amplitude of excitations and temperature ratios are performed for this configuration.

Case R5: A premixed laminar flame with the same jet temperature as that in Case 1, surrounded by a far-field with the same temperature as Case 1. In this case, only the region surrounding the flame's reaction zone approaches the flame's adiabatic temperature.

Cases NR2, NR3, R4a and R5 feature the same peak temperature differences, inferring that the magnitude of entropic and density disturbances in all these cases are similar. However, only cases R4a and R5 feature combustion. Comparison of these four cases should therefore determine the significance of heat release as a source of sound, relative to entropic and other sound sources. Of the two combusting test cases, case R5 is the most physical arrangement. However, this study shows that the significant features of sound production by the two combusting flows (Cases R4 and R5) are similar. 


\section{Numerical solution of governing equations}

There are several challenges in the simulation of sound generation from a jet flow. The source region in the near field must be properly resolved and the computational domain must be sufficiently large to examine sound in the far-field. Boundary conditions must be carefully constructed to prevent spurious reflections. The solver used in this study is a modified version of NTmix [16]. This code has been employed in numerous studies of DNS and LES of non-reacting [e.g. 13, 14] and reacting flows [e.g. 1, 3, 4, 46, 54, 55]. Spatial derivatives are approximated with a 6th order compact finite difference method. Integration in time is achieved with a fully-coupled third-order Runge-Kutta method.

\subsection{Governing equations}

A two-dimensional (2D) computational domain is used. Assuming a one-step, irreversible chemical reaction, the governing equations may written:

$$
\begin{gathered}
\frac{\partial \rho}{\partial t}+\frac{\partial \rho u_{i}}{\partial x_{i}}=0 \\
\frac{\partial \rho u_{i}}{\partial t}+\frac{\partial \rho u_{i} u_{j}}{\partial x_{j}}+\frac{\partial p}{\partial x_{i}}=\frac{\partial \tau_{i j}}{\partial x_{j}} \\
\frac{\partial \rho e_{t}}{\partial t}+\frac{\partial}{\partial x_{i}}\left[\left(\rho e_{t}+p\right) u_{i}\right]=\frac{\partial}{\partial x_{j}}\left(u_{i} \tau_{i j}\right)-\frac{\partial q_{i}}{\partial x_{i}}+Q \dot{\Omega},
\end{gathered}
$$

and

$$
\frac{\partial \rho Y}{\partial t}+\frac{\partial \rho Y u_{i}}{\partial x_{i}}=\frac{\partial}{\partial x_{i}}\left(\rho D \frac{\partial Y}{\partial x_{i}}\right)-\dot{\Omega}
$$

where $\rho$ is the density, $u$ is the velocity, $p$ is the pressure, $x$ is the spatial coordinate, $t$ is time, $\tau$ is the viscous stress tensor, $q$ is the heat flux, $e_{t}$ is the specific internal energy (including kinetic and sensible energy), $Y$ is the fuel mass fraction, $\dot{\Omega}$ is the reaction rate, $D$ is the mass diffusion coefficient, $Q$ is the specific enthalpy of reaction. The pressure, specific internal energy, heat-flux and stress tensors are given by the following constitutive relationships.

$$
\begin{gathered}
p=\frac{\gamma-1}{\gamma} \rho c_{p} T \\
e_{t}=\frac{1}{2} \sum_{k=1}^{2} u_{k}^{2}+\frac{p}{\rho(\gamma-1)},
\end{gathered}
$$

and

$$
\tau_{i j}=\mu\left(\frac{\partial u_{i}}{\partial x_{j}}+\frac{\partial u_{j}}{\partial x_{i}}-\frac{2}{3} \frac{\partial u_{k}}{\partial x_{k}} \delta_{i j}\right) .
$$

The heat-release coefficient in equation 3 is given by

$$
Q=\frac{\alpha}{1-\alpha} c_{p} T_{u}
$$

The reaction rate is expressed as

$$
\dot{\Omega}=\Lambda \rho Y \exp \left[\frac{-\beta(1-\Theta)}{1-\alpha(1-\Theta)}\right],
$$


Table 1: Jet configurations studied.

\begin{tabular}{llccccc}
\hline Case & Configuration & $\begin{array}{c}\text { BC } \\
\text { type }\end{array}$ & $u_{i n} / c_{u}$ & $\begin{array}{c}\text { Amplitude } \\
a\end{array}$ & $R e$ & $\begin{array}{c}\text { Temperature ratio } \\
T_{j} / T_{\infty}\end{array}$ \\
\hline \hline NR1 & Isothermal jet & I & 0.04 & 0.25 & 80 & 1 \\
NR2 & Cold jet & II & 0.04 & 0.25 & 80 & 0.25 \\
NR3 & Hot jet & III & 0.04 & 0.25 & 80 & 4 \\
R4a & Laminar flame & IV & 0.04 & 0.25 & 80 & 0.25 \\
R4b & Laminar flame & IV & 0.08 & 0.25 & 160 & 0.25 \\
R4c & Laminar flame & IV & 0.08 & 0.1 & 160 & 0.25 \\
R4d & Laminar flame & IV & 0.08 & 0.25 & 160 & 0.14 \\
R4e & Laminar flame & IV & 0.08 & 0.25 & 320 & 0.25 \\
R5 & Laminar flame & V & 0.04 & 0.25 & 80 & 1
\end{tabular}

where

$$
\Theta=\left(T-T_{u}\right) /\left(T_{b}-T_{u}\right) \quad \text { and } \quad \Lambda=\mathrm{B}_{0} \exp \left(-\frac{\beta}{\alpha}\right) .
$$

In the above, the variable $B_{0}$ is the pre-exponential factor, $c_{p}$ is the specific heat constant, $\Theta$ is a nondimensional temperature and $T$ is the temperature. The subscripts $u$ and $b$ correspond to unburned and burnt gases, respectively. The heat release parameter $\alpha$ and the Zel'dovich number $\beta$ are given by [45]:

$$
\alpha=\frac{T_{b}-T_{u}}{T_{b}} \text { and } \quad \frac{\beta}{\alpha}=\frac{\mathrm{T}_{\mathrm{a}_{1}}}{\mathrm{~T}_{\mathrm{b}}}
$$

where $T_{a_{1}}$ is the activation temperature. The variable $\beta$ is 8 in this study.

The runs are specified according to parameters as follows:

$$
\begin{gathered}
R e=\frac{\rho_{u} u_{i n} L_{r e f}}{\mu}, \\
P r=\frac{c_{p} \mu}{k}=0.75, \\
S c=\frac{\mu}{\rho_{u} D}=0.75, \\
D a=\frac{D \Lambda}{S_{L}^{2}}, \quad \text { and } \\
S_{L}^{*}=\frac{S_{L}}{c_{u}}=0.01 .
\end{gathered}
$$

In the above, $S_{L}$ represents the laminar flame speed and the viscosity is $\mu$. The Mach number based on $S_{L}$ is denoted $S_{L}^{*}, S c$ is the Schmidt number, $\operatorname{Pr}$ is the Prandtl number and $R e$ is the Reynolds number defined based on the inlet velocity at the centreline $u_{i n}$ and the jet half-width $L_{r e f}$ at the inflow boundary. The Damköhler number is $D a$ and is 238 for Case R4c and 130 for the other combusting cases. All the variables used in the above non-dimensional parameters are constant.

Table 1 summarises the simulation parameters for each configuration. Cases R4a-e feature different combinations of jet Mach number, forcing amplitude, Reynolds number and temperature ratio. 


\subsection{Computational domain and grid parameters}

Figure 2 shows a schematic of the configuration considered in this paper. A symmetry boundary condition was applied on the jet centre-line to reduce computational effort. Non-reflecting boundary conditions were used for the outflow boundaries $[39,58]$. The size of the computational domain was chosen so that the far-field sound was captured accurately. The far field is defined as the part of the computational domain in which the right hand side of Lighthill's acoustic analogy can be approximated as zero. It was found that $y / L_{r e f}>10$ satisfies this criterion for all cases studied, which is consistent with the literature [e.g. 2]. The discretisation of the domain employed 1021 grid points in the streamwise direction from $x=0$ to $32 L_{\text {ref }}$ and 541 grid points in the transverse direction from $y=0$ to $40 L_{r e f}$. Algebraic grid stretching was applied in order to achieve a sufficiently fine resolution of the flame and simultaneously capture far-field sound without excessive computational effort. The local grid stretching was less than $2.0 \%$ between adjacent cells.

Resolution was chosen based on extensive studies of the sensitivity of results to the chosen grid. As an example, a comparison between two different grid sizes, i.e. $801 \times 321$ and $401 \times 161$, for a small domain, i.e. $12 L_{r e f} \times 4 L_{r e f}$, is shown in Figure 3. This figure shows iso-contours of the dilatation $\nabla \cdot \boldsymbol{u}$ and unburnt mass fraction field at one instant for $S t=0.025$ using two grid sizes. By combining the equations for energy and momentum, it may be shown that in the far field, the relationship between the pressure and dilatation is:

$$
\frac{\partial p}{\partial t}+\rho_{\infty} c_{\infty}^{2} \frac{\partial u_{i}}{\partial x_{i}}=0
$$

Acoustic studies of non-reacting flows commonly use dilatation as a marker of sound [e.g. 12]. It also amplifies the high frequency component of pressure such that the numerical noise can be easily observed (see Figure 3). In the context of a premixed flame, dilatation has the useful feature that the flame location is also visible as a high dilatation region.

As can be seen, numerical noise is observed for the coarse grid even though the flame is well resolved in both cases. Figure 4 shows the effect of this numerical error on the pressure history for three different grids, i.e. $401 \times 161,801 \times 321$ and $1201 \times 481$ at $x / L_{r e f}=6$ and $y / L_{\text {ref }}=2$ which is outside the flame. It can be seen that the coarse grid is able to capture the major features of the acoustic field, but with the spurious, high frequency component seen in Figure 3 again apparent. Thus, the grid resolution required for modelling the sound is greater than that required for modelling the flame only.

\subsection{Boundary conditions}

The Navier-Stokes Characteristic Boundary Conditions (NSCBC) were first introduced by Poinsot \& Lele [46]. The present study includes more recent developments of the NSCBC approach, namely, the effect of multi-dimensionality $[58,59]$ and the proper treatment of edges and corners [39].

\subsubsection{Inflow boundary}

The temperature, velocity and unburnt mass fraction are imposed at the inflow boundary. Tables 2 and 3 show the different types of inflow boundary conditions for all cases presented in Table 1 where $y^{*}=y / L_{\text {ref }}$. 
Table 2: Temperature profiles at the inflow boundary.

\begin{tabular}{lc}
\hline Type & Temperature \\
\hline \hline I & $T\left(y^{*}\right)=T_{\text {ref }}$ \\
II & $T\left(y^{*}\right)=\frac{T_{b}+T_{u}}{2}+\frac{T_{b}-T_{u}}{2} \tanh \left[2.5\left(y^{*}-1 / y^{*}\right)\right]$ \\
III & $T\left(y^{*}\right)=\frac{T_{b}+T_{u}}{2}-\frac{T_{b}-T_{u}}{2} \tanh \left[2.5\left(y^{*}-1 / y^{*}\right)\right]$ \\
IV & $T\left(y^{*}\right)=\frac{T_{b}+T_{u}}{2}+\frac{T_{b}-T_{u}}{2} \tanh \left[2.5\left(y^{*}-1 / y^{*}\right)\right]$ \\
V & $T\left(y^{*}\right)=T_{u}+\left(T_{b}-T_{u}\right) \exp \left[-20\left(y^{*}-1.2\right)^{2}\right]$
\end{tabular}

Table 3: Unburnt mass fraction profiles at the inflow boundary.

\begin{tabular}{lc}
\hline Type & Unburnt mass fraction \\
\hline \hline I & $Y\left(y^{*}\right)=0$ (non-reacting) \\
II & $Y\left(y^{*}\right)=0$ (non-reacting) \\
III & $Y\left(y^{*}\right)=0$ (non-reacting) \\
IV & $Y\left(y^{*}\right)=\frac{1}{2}-\frac{1}{2} \tanh \left[2.5\left(y^{*}-1 / y^{*}\right)\right]$ \\
V & $Y\left(y^{*}\right)=\frac{1}{2}-\frac{1}{2} \tanh \left[2.5\left(y^{*}-1 / y^{*}\right)\right]$
\end{tabular}

The inlet velocity is imposed as follows:

$$
u_{1}\left(0, y^{*}, t\right)=u_{0}\left(y^{*}\right)(1+a \sin (2 \pi f t)) \quad \text { and } \quad u_{2}\left(0, y^{*}\right)=0
$$

where

$$
u_{0}\left(y^{*}\right)=\frac{u_{i n}}{2}\left(1-\tanh \left[2.5\left(y^{*}-1 / y^{*}\right)\right]\right)
$$

The variable $u_{i n}$ is the centreline velocity at the inflow boundary. The amplitude of the forcing $a$ as shown in Table 1 was constant. The forcing amplitude of $25 \%$ is used in all cases except for Case R4c to obtain a reasonable amount of flame wrinkling and sound generation by these laminar flames. To investigate the effect of forcing amplitude, Case R4c with an excitation amplitude of $10 \%$ was also considered. The frequency of the inflow forcing $f_{0}$ is represented nondimensionally as a Strouhal number by

$$
S t=f_{0} L_{r e f} / c_{u} .
$$

The jet half-width at the inflow boundary $\left(L_{r e f}\right)$ was used to define the Strouhal number. A similar definition is used in other studies on sound generation by jet flows [e.g. 2]. The paper considers the cases of Strouhal numbers of 0.02, 0.025, 0.05, 0.1 and 1 .

In order to enforce these boundary conditions at the inflow, the time-derivative of temperature, unburnt mass fraction and velocity components can be found as follows,

$$
\begin{gathered}
\frac{\partial T}{\partial t}=\frac{\partial Y}{\partial t}=0 \\
\left.\frac{\partial u_{1}}{\partial t}\right|_{x=0}=2 a \pi f \cos (2 \pi f t) u_{0}\left(y^{*}\right), \text { and }\left.\frac{\partial u_{2}}{\partial t}\right|_{x=0}=0
\end{gathered}
$$


By substituting equations 16 and 17 into NSCBC equations (see Poinsot \& Lele [46]), the characteristic wave amplitudes can be obtained. These wave amplitudes can be used to calculate the time-derivative of density at the inflow boundary. The pressure at the inflow is then calculated from equation 5 .

\section{Solution of Lighthill's acoustic analogy}

Lighthill's equation in pressure form is used to study the sound generation [17],

$$
\frac{1}{c_{\infty}^{2}} \frac{\partial^{2} p}{\partial t^{2}}-\nabla^{2} p=\frac{\partial}{\partial x_{i} \partial x_{j}}\left(\rho u_{i} u_{j}-\tau_{i j}\right)-\frac{\partial^{2} \rho_{e}}{\partial t^{2}},
$$

In the above, variables in the far field are denoted by a subscript $\infty$. The excess density is denoted:

$$
\rho_{e}=\rho-\rho_{\infty}-\left(p-p_{\infty}\right) / c_{\infty}^{2} .
$$

By taking the Fourier transform of equation 18, Lighthill's acoustic analogy can be expressed as a Helmholtz equation,

$$
\left(\omega^{2} / c_{\infty}^{2}+\nabla^{2}\right) \hat{p}=-\frac{\partial}{\partial x_{i} \partial x_{j}}\left(\widehat{\rho u_{i} u_{j}}-\widehat{\tau_{i j}}\right)-\omega^{2} \hat{\rho_{e}}
$$

where $(\hat{)}$ denotes the Fourier transform of () , for example

$$
\hat{p}(x, y, \omega)=\int_{-\infty}^{\infty} p(x, y, t) e^{-i \omega t} d t
$$

Solution of equation 20 for an unbounded domain can be performed using free space Green's functions. Such solutions have been proposed in the literature [e.g. 15, 51]. However, close agreement with such a solution is not possible in the present study for two reasons. First, sound radiation from the forced inflow boundary is significant, and thus the flow cannot be approximated as unbounded. Further, even if this could be ignored, the size of the numerical domain required to give close agreement with the free space results would be significantly larger than that used in some cases.

This paper therefore takes an alternative approach, by developing a solution of equation 20 for a bounded domain. This solution is inevitably more complex than its free space equivalent, which is also discussed briefly in this paper. However, it can be validated against the numerical simulations, thus giving confidence in subsequent analysis of individual acoustic source terms.

Our formulation differs from other approaches such as Ffowcs Williams - Hawkings (FWH) [21] in which the effect of boundaries appears as source terms. In the standard FWH formulation and its variants [41,42], the information on the surface enclosing the source region is used to calculate the density fluctuations in the far field. However, our formulation is preferred in the present study since it allows separation of the sound generated by the near field from that radiated from the inflow boundary.

The bounded domain Green's function solution of equation 20 for a $2 \mathrm{D}$ problem can be expressed as,

$$
\hat{p}\left(\mathbf{r}_{\mathbf{0}}, \omega\right)=\int_{A_{0}} f(\mathbf{r}) G\left(\mathbf{r} \mid \mathbf{r}_{\mathbf{0}}\right) d A-\int_{l_{0}} \hat{p}(\mathbf{r}, \omega) \nabla G\left(\mathbf{r} \mid \mathbf{r}_{\mathbf{0}}\right) \cdot \mathbf{n} d l+\int_{l_{0}} G\left(\mathbf{r} \mid \mathbf{r}_{\mathbf{0}}\right) \boldsymbol{\nabla} \hat{p}(\mathbf{r}, \omega) . \mathbf{n} d l,
$$


where

$$
f(\mathbf{r})=\frac{\partial}{\partial x_{i} \partial x_{j}}\left(\widehat{\rho u_{i} u_{j}}-\widehat{\tau_{i j}}\right)+\omega^{2} \hat{\rho_{e}}
$$

and $G$ is the Green's function. The terms $A_{0}$ and $l_{0}$ are the area and boundary of the source region. If the boundaries have no reflections or acoustic energy flux, the corresponding integral terms will be zero. However, when the flow is excited at the inflow or the boundary passes through the source region, these terms are non-zero and must be retained.

The Green's function for a 2D problem can be expressed using a Hankel function [20],

$$
G\left(\mathbf{r} \mid \mathbf{r}_{\mathbf{0}}\right)=\frac{i}{4} H_{0}^{(1)}\left(\kappa\left|\mathbf{r}-\mathbf{r}_{\mathbf{0}}\right|\right)
$$

where

$$
\kappa=\omega / c_{\infty}
$$

Using equation 24 for the 2D problem shown in Figure 5, equation 22 can be reformulated as follows:

$$
\begin{aligned}
\hat{p}\left(\mathbf{r}_{\mathbf{0}}, \omega\right) & =\underbrace{\frac{i}{4} \int_{A_{0}} f(\mathbf{r}) H_{0}^{(1)}\left(\kappa\left|\mathbf{r}-\mathbf{r}_{\mathbf{0}}\right|\right) d A}_{\text {Term I }} \\
& +\underbrace{\frac{i \kappa}{4} \int_{l_{0}} \hat{p}(\mathbf{r}, \omega) \frac{\partial\left|\mathbf{r}-\mathbf{r}_{\mathbf{0}}\right|}{\partial x_{i}} H_{1}^{(1)}\left(\kappa\left|\mathbf{r}-\mathbf{r}_{\mathbf{0}}\right|\right) \cdot \mathbf{n} d l}_{\text {Term II }} \\
& +\underbrace{\frac{i}{4} \int_{l_{0}} H_{0}^{(1)}\left(\kappa\left|\mathbf{r}-\mathbf{r}_{\mathbf{0}}\right|\right) \frac{\partial \hat{p}}{\partial x_{i}}(\mathbf{r}, \omega) \cdot \mathbf{n} d l}_{\text {Term III }} .
\end{aligned}
$$

One needs to distinguish between the variable $\hat{p}$ on the right and left hand sides of equation 26. The variable $\hat{p}$ on the right hand side is obtained from the simulations since it is set as the boundary condition in Lighthill's equation, while $\hat{p}$ on the left hand side is the solution of Lighthill's equation. Term I in equation 26 shows the contribution of the right hand side of Lighthill's equation to the radiated sound. Terms II and III show the effect of reflection, excitation and non-zero sources at the boundaries. In order to obtain the Fourier transform of the pressure in the far field, terms II and III must be calculated at all boundaries of the computational domain. Boundary of the domain, $l_{0}$ is therefore decomposed into four lines, i.e. two horizontal lines at $-y_{l}$ and $+y_{l}$ extending from $x=0$ to $x=x_{l}$ and two vertical lines at 0 and $x_{l}$, extending from $y=-y_{l}$ to $y=+y_{l}$. Terms II and III may be then expressed as, 


$$
\begin{aligned}
\int_{l_{0}} \hat{p}(\mathbf{r}, \omega) \frac{\partial\left|\mathbf{r}-\mathbf{r}_{\mathbf{0}}\right|}{\partial x_{i}} H_{1}^{(1)}\left(\kappa\left|\mathbf{r}-\mathbf{r}_{\mathbf{0}}\right|\right) \cdot \mathbf{n} d l & =\left.\int_{-y_{l}}^{y_{l}} \hat{p}(\mathbf{r}, \omega)\right|_{x=0} \frac{x_{\mathbf{r}_{\mathbf{0}}}}{r_{1}} H_{1}^{(1)}\left(\kappa r_{1}\right) d y \\
& +\left.\int_{-y_{l}}^{y_{l}} \hat{p}(\mathbf{r}, \omega)\right|_{x=x_{l}} \frac{\left(x_{l}-x_{\mathbf{r}_{\mathbf{0}}}\right)}{r_{2}} H_{1}^{(1)}\left(\kappa r_{2}\right) d y \\
& +\left.\int_{0}^{x_{l}} \hat{p}(\mathbf{r}, \omega)\right|_{y=y_{l}} \frac{y_{l}-y_{\mathbf{r}_{\mathbf{o}}}}{r_{3}} H_{1}^{(1)}\left(\kappa r_{3}\right) d x \\
& +\left.\int_{0}^{x_{l}} \hat{p}(\mathbf{r}, \omega)\right|_{y=-y_{l}} \frac{y_{l}+y_{\mathbf{r}_{0}}}{r_{4}} H_{1}^{(1)}\left(\kappa r_{4}\right) d x
\end{aligned}
$$

and

$$
\begin{aligned}
\int_{l_{0}} H_{0}^{(1)}\left(\kappa\left|\mathbf{r}-\mathbf{r}_{\mathbf{0}}\right|\right) \frac{\partial \hat{p}}{\partial x_{i}}(\mathbf{r}, \omega) . \mathbf{n} d l= & -\left.\int_{-y_{l}}^{y_{l}} \frac{\partial \hat{p}}{\partial x}(\mathbf{r}, \omega)\right|_{x=0} H_{0}^{(1)}\left(\kappa r_{1}\right) d y \\
& +\left.\int_{-y_{l}}^{y_{l}} \frac{\partial \hat{p}}{\partial x}(\mathbf{r}, \omega)\right|_{x=x_{l}} H_{0}^{(1)}\left(\kappa r_{2}\right) d y \\
& +\left.\int_{0}^{x_{l}} \frac{\partial \hat{p}}{\partial y}(\mathbf{r}, \omega)\right|_{y=y_{l}} H_{0}^{(1)}\left(\kappa r_{3}\right) d x \\
& -\left.\int_{0}^{x_{l}} \frac{\partial \hat{p}}{\partial y}(\mathbf{r}, \omega)\right|_{y=-y_{l}} H_{0}^{(1)}\left(\kappa r_{4}\right) d x
\end{aligned}
$$

The variables $r_{1}, r_{2}, r_{3}$ and $r_{4}$ (see Figure 5) are defined as

$$
\begin{aligned}
& r_{1}=\sqrt{x_{0}^{2}+\left(y_{0}-y^{\prime}\right)^{2}}, \\
& r_{2}=\sqrt{\left(x_{0}-x_{l}\right)^{2}+\left(y_{0}-y^{\prime}\right)^{2}}, \\
& r_{3}=\sqrt{\left(x_{0}-x^{\prime}\right)^{2}+\left(y_{0}-y_{l}\right)^{2}}, \text { and } \\
& r_{4}=\sqrt{\left(x_{0}-x^{\prime}\right)^{2}+\left(y_{0}+y_{l}\right)^{2}} .
\end{aligned}
$$

where $x^{\prime}$ varies from 0 to $x_{l}$ and $y^{\prime}$ varies from $-y_{l}$ to $y_{l}$. It is straightforward to show that if the boundary at $x=x_{l}$ does not pass through the source region and is a non-reflecting outflow boundary, the sum of terms II and III calculated at $x=x_{l}, y=-y_{l}$ and $y=y_{l}$ can be neglected, resulting in equation 26 reducing to

$$
\begin{aligned}
\hat{p}\left(\mathbf{r}_{\mathbf{0}}, \omega\right) & =\frac{i}{4} \int_{A_{0}} f(\mathbf{r}) H_{0}^{(1)}\left(\kappa\left|\mathbf{r}-\mathbf{r}_{\mathbf{0}}\right|\right) d A \\
& +\left.\frac{i \kappa}{4} \int_{-y_{l}}^{y_{l}} \hat{p}(\mathbf{r}, \omega)\right|_{x=0} \frac{x_{\mathbf{r}_{0}}}{r_{1}} H_{1}^{(1)}\left(\kappa r_{1}\right) d y-\left.\frac{i}{4} \int_{-y_{l}}^{y_{l}} \frac{\partial \hat{p}}{\partial x}(\mathbf{r}, \omega)\right|_{x=0} H_{0}^{(1)}\left(\kappa r_{1}\right) d y .
\end{aligned}
$$

Equation 29 can be simplified further by dividing the problem into two sub-problems, $a$ and $b$, each of which is a solution of equation 20 such that

$$
\hat{p}\left(\mathbf{r}_{\mathbf{0}}, \omega\right)=\hat{p_{a}}\left(\mathbf{r}_{\mathbf{0}}, \omega\right)+\hat{p_{b}}\left(\mathbf{r}_{\mathbf{0}}, \omega\right),
$$


where

$$
f_{a}(\mathbf{r})=f(\mathbf{r}),\left.\quad \hat{p_{a}}\right|_{x=0}=\left.\hat{p}\right|_{x=0},\left.\quad \frac{\partial \hat{p_{a}}}{\partial x}\right|_{x=0}=0
$$

and

$$
f_{b}(\mathbf{r})=0,\left.\quad \hat{p_{b}}\right|_{x=0}=0,\left.\quad \frac{\partial \hat{p_{b}}}{\partial x}\right|_{x=0}=\left.\frac{\partial \hat{p}}{\partial x}\right|_{x=0} .
$$

Equation 29 then shows that the solution for problem $a$ is

$$
\hat{p_{a}}\left(\mathbf{r}_{\mathbf{0}}, \omega\right)=\frac{i}{4} \int_{A_{0}} f(\mathbf{r}) H_{0}^{(1)}\left(\kappa\left|\mathbf{r}-\mathbf{r}_{\mathbf{0}}\right|\right) d A+\left.\frac{i \kappa}{4} \int_{-y_{l}}^{y_{l}} \hat{p}(\mathbf{r}, \omega)\right|_{x=0} \frac{x_{\mathbf{r}_{\mathbf{0}}}}{r_{1}} H_{1}^{(1)}\left(\kappa r_{1}\right) d y,
$$

whilst the solution for problem $b$ is

$$
\hat{p_{b}}\left(\mathbf{r}_{\mathbf{0}}, \omega\right)=-\left.\frac{i}{4} \int_{-y_{l}}^{y_{l}} \frac{\partial \hat{p}}{\partial x}(\mathbf{r}, \omega)\right|_{x=0} H_{0}^{(1)}\left(\kappa r_{1}\right) d y .
$$

Since $\partial \hat{p_{a}} / \partial x=0$ in problem $a$, its solution is symmetric around $x=0$. The method of images can therefore be used [20], resulting in

$$
\hat{p_{a}}\left(\mathbf{r}_{\mathbf{0}}, \omega\right)=\frac{i}{4} \int_{A_{0}} f(\mathbf{r}) H_{0}^{(1)}\left(\kappa\left|\mathbf{r}-\mathbf{r}_{\mathbf{0}}\right|\right) d A+\frac{i}{4} \int_{A_{0}} f(\mathbf{r}) H_{0}^{(1)}\left(\kappa\left|\mathbf{r}^{\prime}-\mathbf{r}_{\mathbf{0}}\right|\right) d A,
$$

where

$$
\boldsymbol{r}^{\prime}=-x \boldsymbol{i}+y \boldsymbol{j} .
$$

The complete solution for $\hat{p}$ can then be obtained as follows.

$$
\begin{aligned}
\hat{p}\left(\mathbf{r}_{\mathbf{0}}, \omega\right) & =\frac{i}{4} \int_{A_{0}} f(\mathbf{r}) H_{0}^{(1)}\left(\kappa\left|\mathbf{r}-\mathbf{r}_{\mathbf{0}}\right|\right) d A+\frac{i}{4} \int_{A_{0}} f(\mathbf{r}) H_{0}^{(1)}\left(\kappa\left|\mathbf{r}^{\prime}-\mathbf{r}_{\mathbf{0}}\right|\right) d A \\
& -\left.\frac{i}{4} \int_{-y_{l}}^{y_{l}} \frac{\partial \hat{p}}{\partial x}(\mathbf{r}, \omega)\right|_{x=0} H_{0}^{(1)}\left(\kappa r_{1}\right) d y .
\end{aligned}
$$

In the present case, the last term in equation 37 is significant within the jet and so must be included in this solution. For example, as shown in Figure 6, $\partial \hat{p} / \partial n$ cannot be neglected in the near field for $S t=1$ and case NR1. The first two terms on the right hand side of equation 37 both contain $f(\mathbf{r})$. These two terms are not combined since one is the result of generation of sound by the source region and the other is the result of reflection from the inflow boundary. The third term can be interpreted as the effect of excitation.

The resulting Fourier transform of the pressure in equation 37 can be considered as a sum of the components,

$$
\hat{p}=\hat{p}_{i n}+\hat{p}_{s t}+\hat{p}_{e x},
$$

where

$$
\begin{aligned}
\hat{p}_{i n}\left(\mathbf{r}_{\mathbf{o}}, \omega\right) & =\frac{i}{4} \int_{A_{0}} f(\mathbf{r}) H_{0}^{(1)}\left(\kappa\left|\mathbf{r}^{\prime}-\mathbf{r}_{\mathbf{o}}\right|\right) d A-\left.\frac{i}{4} \int_{-y_{l}}^{y_{l}} \frac{\partial \hat{p}}{\partial x}(\mathbf{r}, \omega)\right|_{x=0} H_{0}^{(1)}\left(\kappa r_{1}\right) d y \\
\hat{p}_{s t}\left(\mathbf{r}_{\mathbf{0}}, \omega\right) & =\frac{i}{4} \int_{A_{0}} \frac{\partial\left(\widehat{\rho u_{i} u_{j}}-\widehat{\tau_{i j}}\right)}{\partial x_{i} \partial x_{j}} H_{0}^{(1)}\left(\kappa\left|\mathbf{r}-\mathbf{r}_{\mathbf{o}}\right|\right) d A
\end{aligned}
$$


and

$$
\hat{p}_{e x}\left(\mathbf{r}_{\mathbf{0}}, \omega\right)=\frac{i \omega^{2}}{4} \int_{A_{0}} \hat{\rho_{e}} H_{0}^{(1)}\left(\kappa\left|\mathbf{r}-\mathbf{r}_{\mathbf{0}}\right|\right) d A
$$

The variables $\hat{p}_{i n}, \hat{p}_{s t}$ and $\hat{p}_{e x}$ describe the respective contribution of the inflow boundary, the part of Lighthill's stress tensor including the Reynolds-stress term and the excess density term to the radiated sound.

\subsection{Solution of Dowling's reformulation of Lighthill's equation}

Dowling [18] rearranged the excess density term $\partial^{2} \rho_{e} / \partial t^{2}$ in Lighthill's equation to examine sound generation by combusting flows,

$$
\begin{aligned}
\frac{1}{c_{\infty}^{2}} \frac{\partial^{2} p}{\partial t^{2}}-\nabla^{2} p & =\frac{\partial^{2}}{\partial x_{i} \partial x_{j}}\left(\rho u_{i} u_{j}-\tau_{i j}\right) \\
& +\frac{\partial}{\partial t}\left[\frac{\rho_{\infty}}{\rho}\left(\frac{Q \dot{\Omega}}{c_{p} T}-\frac{1}{c_{p} T} \frac{\partial q_{i}}{\partial x_{i}}+\frac{\tau_{i j}}{c_{p} T} \frac{\partial u_{i}}{\partial x_{j}}\right)\right] \\
& +\frac{1}{c_{\infty}^{2}} \frac{\partial}{\partial t}\left[\left(1-\frac{\rho_{\infty} c_{\infty}^{2}}{\rho c^{2}}\right) \frac{D p}{D t}-\frac{p-p_{\infty}}{\rho} \frac{D \rho}{D t}\right] \\
& +\frac{\partial^{2}}{\partial x_{i} \partial t}\left(u_{i} \rho_{e}\right) .
\end{aligned}
$$

The term in the top row on the right hand side of equation 42 is the well known quadrupole source of Lighthill's theory. All other terms in the remaining rows are part of the excess density term. The term in the second row on the right hand side of equation 42 is a monopolar source term. It comprises the effects of heat release, heat conduction and viscous work on the radiated sound. The term in the third row in equation 42, as interpreted by Dowling [18], is appreciable if the mean density and sound speed of the source region are different from the far field. This term can be reformulated as follows:

$$
\frac{1}{c_{\infty}^{2}} \frac{\partial}{\partial t}\left[\left(1-\frac{\rho_{\infty} c_{\infty}^{2}}{\rho c^{2}}\right) \frac{D p}{D t}-\frac{p-p_{\infty}}{\rho} \frac{D \rho}{D t}\right]=\frac{1}{c_{\infty}^{2}}\left(\frac{p^{\prime}}{p}\right)\left(\frac{D p}{D t}-\frac{c^{2}}{\gamma} \frac{D \rho}{D t}\right)
$$

For small $p^{\prime} / p$, as occurs in low Mach number flows, this term should be small compared to the other source terms. The last term in equation 42 is a dipole source and describes the effect of changes in the momentum of density inhomogeneities.

The variable $\hat{p}_{e x}$ in equation 38 can be reformulated using equation 42 ,

$$
\hat{p}_{e x}=\hat{p}_{h r}+\hat{p}_{t h}+\hat{p}_{v i s}+\hat{p}_{e x 1}+\hat{p}_{e x 2},
$$


where

$$
\begin{aligned}
& \hat{p}_{h r}\left(\mathbf{r}_{\mathbf{0}}, \omega\right)=-\frac{\omega \rho_{\infty} Q}{4} \int_{A_{0}}\left(\widehat{\frac{\dot{\Omega}}{\rho c_{p} T}}\right) H_{0}^{(1)}\left(\kappa\left|\mathbf{r}-\mathbf{r}_{\mathbf{0}}\right|\right) d A \\
& \hat{p}_{t h}\left(\mathbf{r}_{\mathbf{0}}, \omega\right)=-\frac{\omega \rho_{\infty}}{4} \int_{A_{0}}\left(\frac{\widehat{\nabla \cdot \boldsymbol{q}}}{\rho c_{p} T}\right) H_{0}^{(1)}\left(\kappa\left|\mathbf{r}-\mathbf{r}_{\mathbf{0}}\right|\right) d A \\
& \hat{p}_{v i s}\left(\mathbf{r}_{\mathbf{0}}, \omega\right)=-\frac{\omega \rho_{\infty}}{4} \int_{A_{0}}\left(\frac{\tau_{i j} \widehat{\partial u_{i} / \partial x_{j}}}{\rho c_{p} T}\right) H_{0}^{(1)}\left(\kappa\left|\mathbf{r}-\mathbf{r}_{\mathbf{0}}\right|\right) d A \\
& \hat{p}_{\text {ex } 1}\left(\mathbf{r}_{\mathbf{0}}, \omega\right)=-\frac{\omega}{4 c_{\infty}^{2}} \int_{A_{0}}\left[\left(1-\frac{\rho_{\infty} c_{\infty}^{2}}{\rho c^{2}}\right) \widehat{\frac{D p}{D t}}-\frac{p-p_{\infty}}{\rho} \frac{D \rho}{D t}\right] H_{0}^{(1)}\left(\kappa\left|\mathbf{r}-\mathbf{r}_{\mathbf{0}}\right|\right) d A
\end{aligned}
$$

and

$$
\hat{p}_{e x 2}\left(\mathbf{r}_{\mathbf{0}}, \omega\right)=-\frac{\omega}{4} \int_{A_{0}} \frac{\partial}{\partial x_{i}} \widehat{\left(u_{i} \rho_{e}\right)} H_{0}^{(1)}\left(\kappa\left|\mathbf{r}-\mathbf{r}_{\mathbf{0}}\right|\right) d A
$$

The variables $\hat{p}_{h r}, \hat{p}_{t h}$ and $\hat{p}_{v i s}$ are the effects of heat release, heat conduction and viscous stress on the radiated sound respectively. Terms $\hat{p}_{\text {ex } 1}$ and $\hat{p}_{\text {ex } 2}$ are due to the effects of density inhomogeneities. As mentioned above, $p_{e x 1}$ is expected to be small for low Mach number flows.

\section{Results and discussion}

\subsection{Simulation results}

\subsubsection{Non-reacting jets}

Figures 7 a-c show the dilatation $\boldsymbol{\nabla} . \boldsymbol{u}$ field at an instant for the non-reacting cases forced at $S t=f L_{r e f} / c_{u}=1$ and $S t=0.1$. Figures $7 \mathrm{a}-\mathrm{c}$ suggest that the jet has a significant effect on the directionality of all non-reacting cases in the high frequency limit. For instance, Figure 7 a shows that the magnitude of the dilatation significantly decreases at an angle $\phi$ greater than $30^{\circ}$ for $S t=1$ where $\phi$ represents the angle from the jet centreline. This is consistent with the observation in Figure 8a (Case NR1). These results suggest that the contribution of the quadrupolar and dipolar source terms in Lighthill's equation should be significant in these instances. As can be seen from Figure 8, for Cases NR2 and NR3 $(S t=1)$ the jet has a significant effect on the incoming acoustic waves. This is due to the refraction of sound waves as a result of a large difference between the speed of sound in the near field and far field. (The speed of sound in the hot region is twice the speed of sound in the cold region.) As shown in Figure 8, a 'zone of silence' $\left(0<\phi<30^{\circ}\right)$ is formed for Case NR3 and $S t=1$ which is the result of a larger sonic velocity in the near field compared with the far field.

At $S t=0.1$, Figures 7a-c show an almost monopolar pattern for the non-reacting cases. This is also evident in Figure $8 \mathrm{~b}$ and may be caused by the strong contribution of the inflow boundary and the monopolar source terms in Dowling's formulation of Lighthill's equation. More details about the magnitude of different terms will be discussed in section 4.2.

Figure 9 shows a comparison of the pressure amplitude versus non-dimensional Strouhal number for cases NR1-3, R4a and R5. As can be seen, the non-reacting cases feature a sharp peak at the excitation frequency. This implies that these cases can be categorised using the frequency of excitation. 


\subsubsection{Combusting jets}

Figures $7 \mathrm{~d}$ and e show the dilatation field at an instant for Cases R4a and R5. Similar to the non-reacting cases, directionality of the acoustic field is observed at the high frequency limit $(S t=1)$. This can also be seen in Figure 8 , showing that the directionality pattern changes at angles of $60^{\circ}$ and $80^{\circ}$ for Cases R4a and R5 respectively. As shown in Figures 8, monopolar behaviour is observed at low to intermediate forcing frequencies. Later analysis will show that this monopolar behaviour is due to both the contribution of the inflow boundary and monopolar source terms in Lighthill's equation.

Figure 10 shows instantaneous contours of unburnt mass fraction at four different forcing frequencies for Cases R4a, R4b and R5. As briefly discussed in [56], qualitatively similar observations have been made in experimental investigations $[6,32,35,36]$. For the highest Strouhal number, the flame does not respond to the inflow forcing (Figure 10a). As Strouhal number is reduced, enhanced corrugation of the flame is observed with fluctuations on the scale of the convective wavelength $u_{i n} / f$ that propagate in the streamwise direction. Figure 10 also shows that the flame dynamics with the hot (Case R4a) and cold far-field (Case R5) are very similar.

Two important phenomena can be observed at several intermediate forcing frequencies in the combusting cases (Figures 10c-e). The first phenomenon will be termed 'flame pinch-off', in which a pocket of reactant is detached from an elongated filament in the flame. This event occurs close to the flame tip and may be observed between the $3^{\text {th }}$ and $4^{\text {th }}$ frames of Figure $10 \mathrm{c}$ for Case R4b and elsewhere. An island of unburned reactants is formed at the flame tip as a result of flame pinch-off and is progressively consumed. This phenomenon will be termed 'flame island burn-out'. Such events have been observed experimentally in other studies [e.g. $32,34]$.

Figure 11 shows dilatation at eight time instants over one period of the inflow forcing for Case R4a with $S t=0.02$ and $u_{i n} / c_{u}=0.04$. Island burn-out and flame pinch-off may be observed as strong sources of sound which are essentially monopolar. The observation may be explained by noting that these annihilation events result in rapid changes of the area of the flame surface and thus the rate of heat release. The phenomena can also be observed in Figure 12 at higher Mach number. For the flame in a cold domain (Case R5, Figure 13), the region of non-uniform temperature affects the directionality of the sound waves, but the mechanisms of sound generation (i.e. pinch-off and island burn-out) are the same.

These results confirm and reinforce experimental observations of similar phenomena. Kidin et al. $[33,34]$ studied the sound produced by an acoustically forced conical flame. Two distinct maxima in the time-history of the far-field pressure were observed. The first peak had the largest amplitude and was identified with the annihilation of a 'flame neck', which is analogous to the 'flame pinch-off' discussed here. The second peak was identified with the burning of a 'pocket' of fresh reactants, which is analogous to the 'island burn-out' discussed here. Using experiments on laminar flames, Candel et al. [8] studied sound prdoduction in three simple situations: interaction of a flame and a wall, flame-vortex interaction, and the mutual annihilation of two flames, and concluded that rapid destruction of flame surface area was the most important source of sound. In a separate study by the same group [48], flame collision events were identified as a strong source of noise in " $\mathrm{M}$ " shaped, laminar perturbed flames.

Comparison of the dilatation fields after pinch-off and island burn-out events (for instance, Figure 12 of $t=0$ and $t=5 T / 8$ ) shows that the pinch-off event is a stronger source of sound compared with the island burn-out event. In the observed pinch-off event, two curved flames merge into one another. In the limit of zero flame curvature, this event can be considered as a collision of two planar flames. The sound generation in a planar case therefore sets one limit 
on the sound generation by flame pinch-off. The island burn-out is similar to an axisymmetric island burn-out event. The physics of these idealisations of the annihilation events has been presented in previous studies $[54,55]$.

The presence of annihilation events has a significant effect on the pressure spectrum. As can be seen in Fig. 9, at $S t=0.02$, a broader range of frequencies compared to the nonreacting cases contribute to sound generation in the far-field. This is because annihilation events introduce new time scales which are different from the period of excitation.

\subsection{Numerical solution of Lighthill's equation}

Equation 37 was solved numerically. Figure 14 shows a comparison between this solution of Lighthill's equation and the simulation results for several cases. The simulation results and numerical solution of Lighthill's equation agree very well in all cases, validating the numerical solution. It can also be observed that the inflow boundary significantly contributes to the solution of Lighthill's equation at $S t=0.02$.

\subsubsection{Contribution of the source terms}

The magnitudes of $\hat{p}_{s t}$ and $\hat{p}_{e x}$ defined in equations 40 and 41 evaluated at $x=8 L_{r e f}$, $y=30 L_{r e f}$ are shown in Figure 15 for several cases. As can be seen, the excess density term is the dominant term in the non-isothermal jets and both flames.

In order to investigate the contribution of the excess density term in more detail, contour plots of this term are shown for cases NR2, NR3, R4a and R5 for high and low frequency excitation in Figure 16. For both cases R4a and R5 and $S t=0.02$, the source region covers the part of the domain where the flame surface area variation occurs. However, when $S t=1$, the flame surface is a small part of the source region.

The magnitudes of $\hat{p}_{h r}, \hat{p}_{t h}, \hat{p}_{v i s c}, \hat{p}_{e x 1}$ and $\hat{p}_{e x 2}$ defined in equations 45-49 are shown in Figures 17 and 18 . The density inhomogeneity term $\hat{p}_{e x 1}$ is small compared with the other terms for all cases, which is the expected behaviour for a low Mach number flow and is consistent with the discussion in section 3.1 (equation 43). The viscous stress term $\hat{p}_{v i s}$ is also negligible for all cases studied here. However, it can be observed that $\hat{p}_{e x 2}$, which represents changes in the momentum of density inhomogeneities, dominates for the non-isothermal, non-reacting cases (i.e. NR2 and NR3). It may also be observed that the term due to heat conduction, $\hat{p}_{t h}$ is at least an order of magnitude smaller than $\hat{p}_{\text {ex } 2}$ for the non-reacting cases.

For the combusting flows (Cases R4 and R5), Figure 18 shows that both $\hat{p}_{h r}$ and $\hat{p}_{e x 2}$ are significant source terms. The relative contribution of the heat release term $\hat{p}_{h r}$ increases as the excitation frequency decreases in most cases. However, the excess density term $\hat{p}_{\text {ex } 2}$ remains the dominant term in some cases for combusting flows. This contradicts the view that the heat release source term is the only significant term in a low Mach number combusting jet. Also, It may be observed that $\hat{p}_{t h}$ is comparable to $\hat{p}_{h r}$ for $S t \geq 0.1$ in Cases R4a and R5. This may be expected since heat conduction and heat release are intimately connected in premixed flames.

Comparison of Cases R4b $(a=0.25)$ and R4c $(a=0.1)$ shows that lowering the forcing amplitude leads to greater contribution from the heat release term relative to the excess density term. However, these two terms are still comparable for Case R4c. Further reduction in the amplitude of excitation from $10 \%$ will lead to less flame wrinking and sound generation as a result. Therefore, amplitudes lower than $10 \%$ were not considered in this study. Increasing the temperature ratio from $T_{b} / T_{u}=4$ (Case R4b) to $T_{b} / T_{u}=7$ (Case R4d) once again shows an increase in the contribution of the heat release term in particular at low frequencies. However, 
the excess density term is still comparable to the heat release term for Case R4d. By comparing Cases R4b $(R e=160)$ and $\mathrm{R} 4 \mathrm{e}(R e=320)$ no clear conclusion can be drawn on the effect of Reynolds number on the relative contribution of the heat release term to the excess density term. This is very likely because of the relatively small range of Re studied.

Nonetheless, comparison of all combusting cases in Figure 18 shows that both the heat release and excess density terms play a significant role in sound generation. Further comparison of Figure 17 and 18 shows that the excess density terms are comparable.

\section{Conclusions}

Acoustically forced laminar jet flows have been studied theoretically and numerically. Isothermal, non-isothermal and combusting jets were compared. The non-combusting, non-isothermal jet flows were simulated with the same temperature ratio as the combusting flows, implying that the magnitude of entropic and density disturbances was similar to the combusting cases.

All cases studied showed a significant directional dependence of the radiated sound on the jet forcing frequency. The sound radiated was directional at high forcing frequency, but monopolar at low forcing frequency in all cases. For the combusting flows, this monopolar behaviour at low frequency was in part due to flame annihilation events, demonstrating the relevance of previous work that considered the one dimensional, limiting cases of planar, cylindrical, and spherical flame annihilations [54, 55].

Lighthill's equation as reformulated by Dowling [18] was then employed to examine the relative importance of the source terms involved in these jet flows. Since the far field was resolved in the simulations, validation of the numerical solution of Lighthill's equation was first performed, providing a solid basis on which to discuss the relative magnitude of different source terms in Lighthill's equation.

As expected, the so-called 'excess density' term in Lighthill's equation was observed to be significantly larger than the 'Reynolds stress term' for all non-combusting, non-isothermal cases. More surprisingly however, decomposition of this excess density term revealed that, in the limit of high forcing frequency, the term describing changes in the momentum of density inhomogeneities was the dominant source term for all combusting and non-isothermal cases. Over the range of frequencies at which flame annihilation occurred, this term due to density inhomogeneities was still comparable in magnitude to the heat-release source term. It therefore appears that a number of terms contribute to sound generation for acoustically forced, laminar combusting flows. Further research is now needed to investigate the contribution of each term in turbulent premixed flames. 
Case NR2

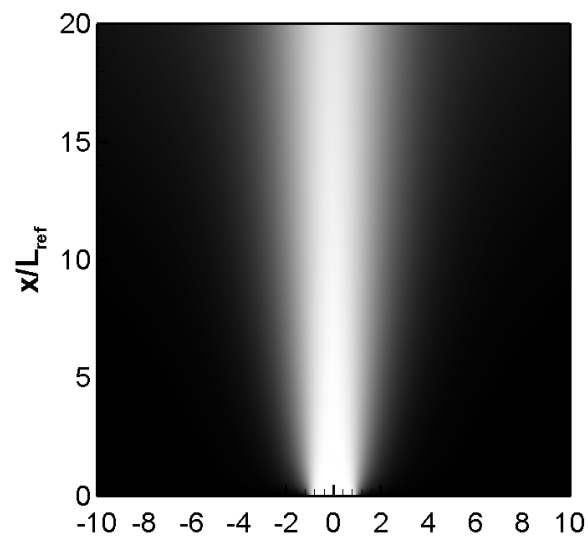

Case R4a

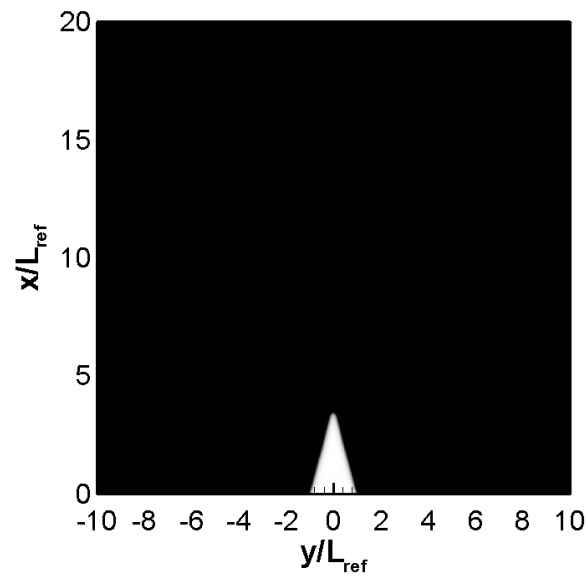

Case NR3

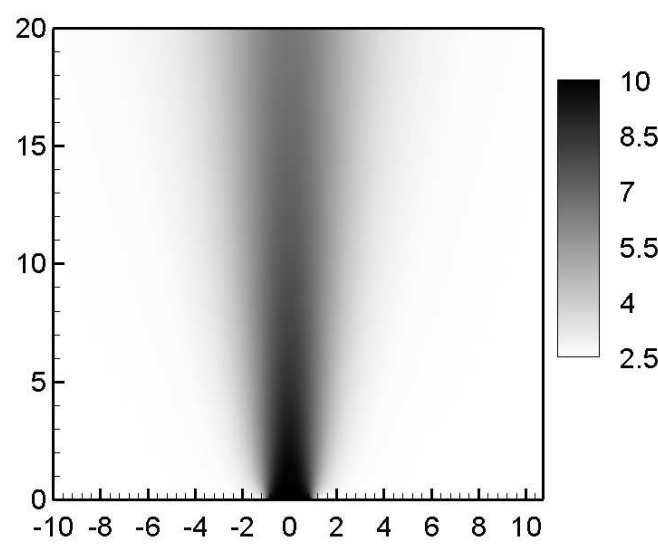

Case R5

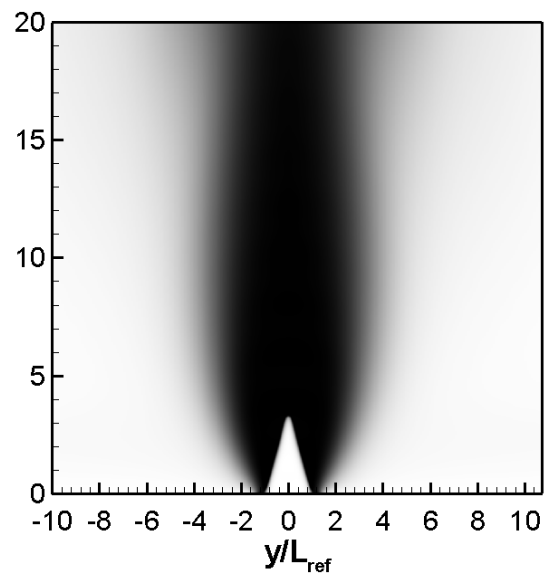

Fig. 1: Steady temperature field $T c_{p} / c_{u}^{2}$ for Cases NR2, NR3, R4a and R5. 
a)

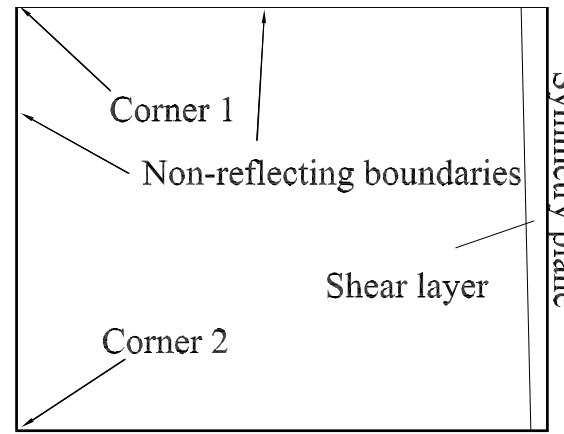

Imposed velocity and temperature

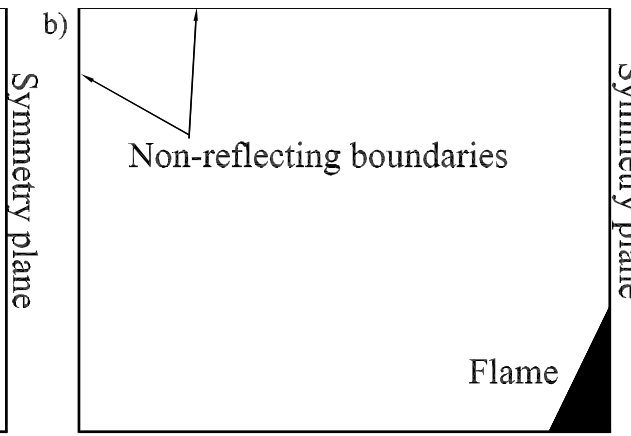

Imposed velocity, temperature

Fig. 2: Schematic of the computational domain: a) Cases NR1-NR3 and b) Cases R4-5 (not to scale). 
Unburnt mass fraction

(a)

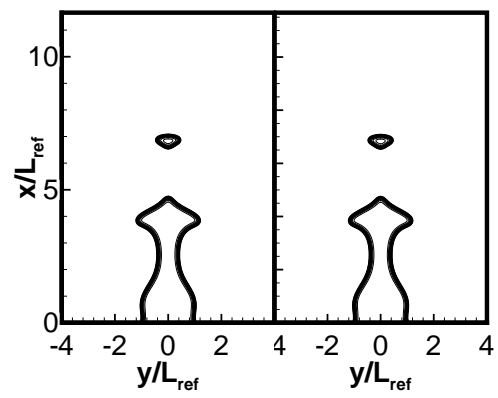

Dilatation

(a)

(b)

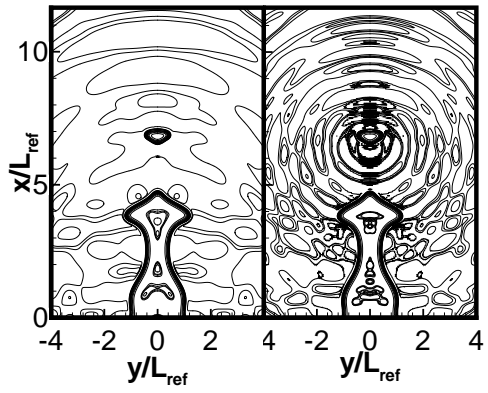

Fig. 3: Unburnt mass fraction and dilatation contours at the same instant for a) fine and b) coarse grids, Case R4b, $u_{i n} / c_{u}=0.08$ and $S t=0.025$. 


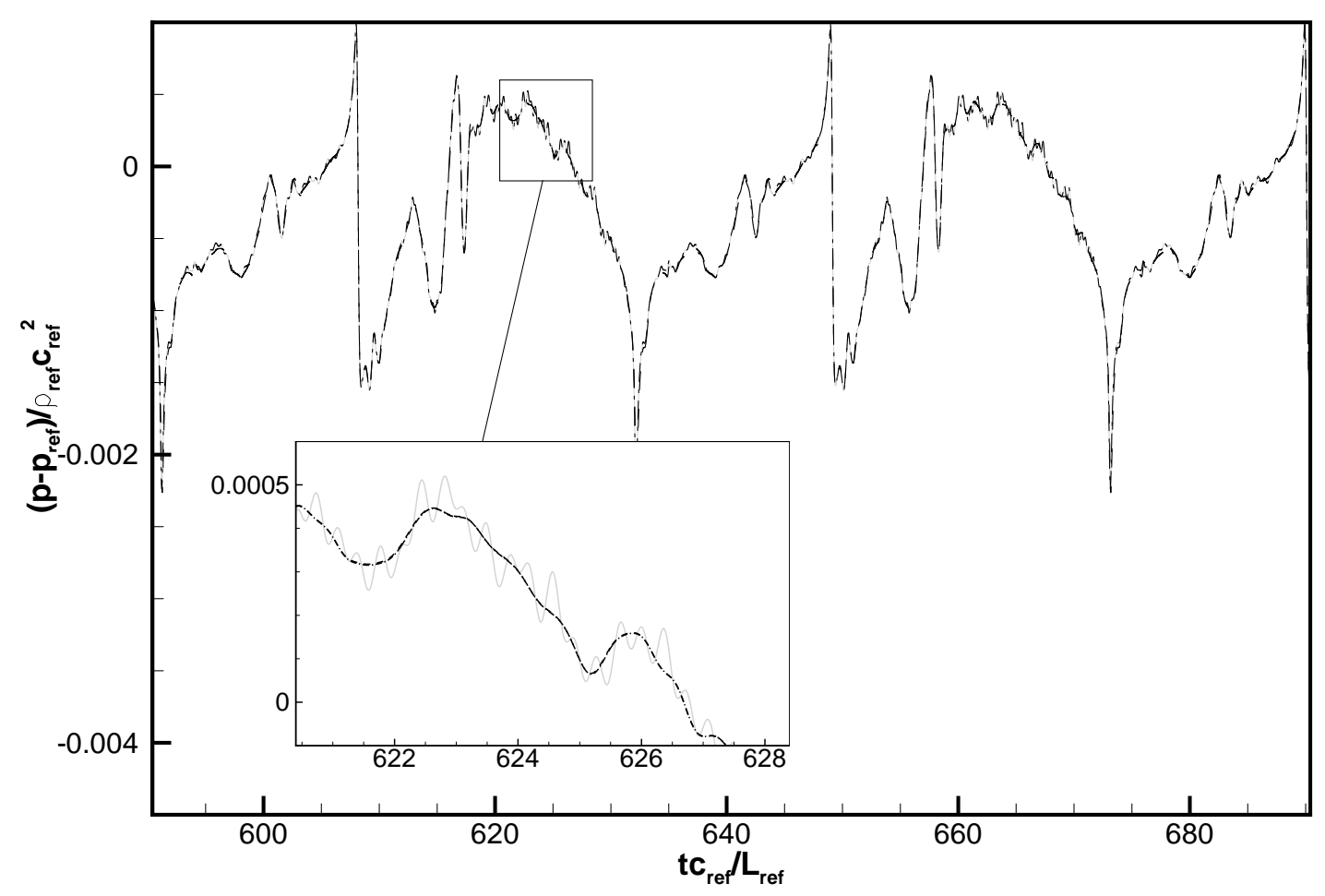

Fig. 4: Pressure fluctuations outside the flame at $x / L_{r e f}=6$ and $y / L_{r e f}=2$ for three different grids: $401 \times 161$ (solid line), $801 \times 321$ (dashed) and $1201 \times 481$ (dash-dot). 


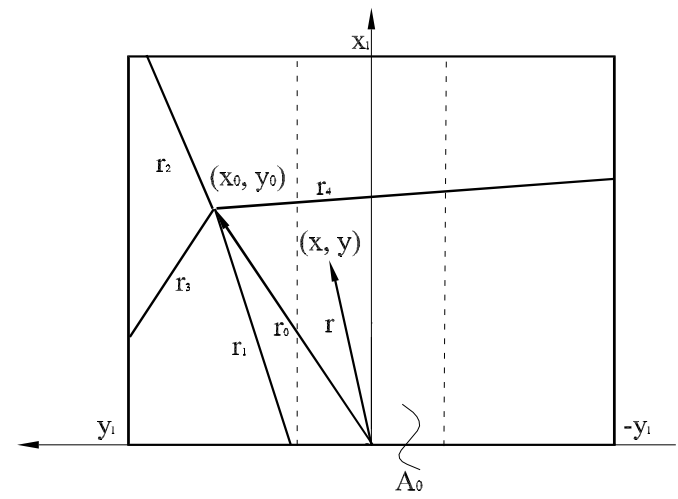

Fig. 5: Schematic of the coordinate system and the source region. Source region shown with $A_{0}$ is a part of the domain within the dashed lines. The vector $\mathbf{r}$ is a distance vector of a point within the source region whereas $\mathbf{r}_{\mathbf{0}}$ is a distance vector for a point in the far field. 


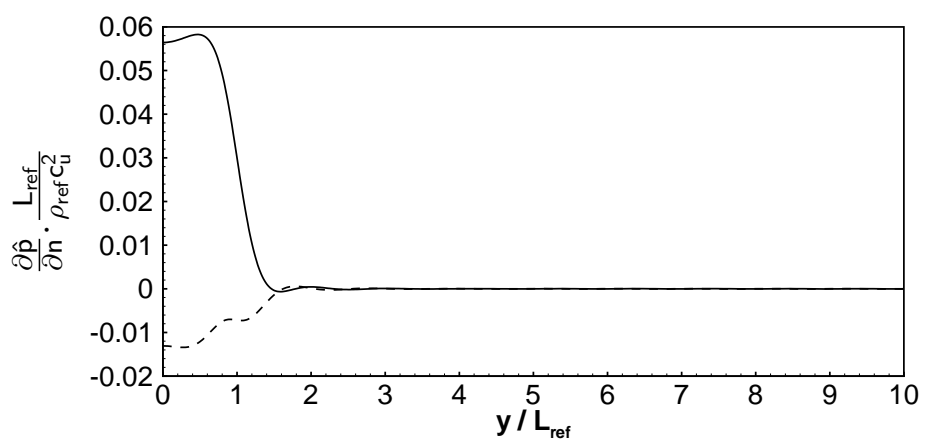

Fig. 6: Real (solid line) and imaginary (dashed line) parts of $\partial \hat{p} / \partial n$ at the inflow boundary for $S t=1$ and Case NR1. 

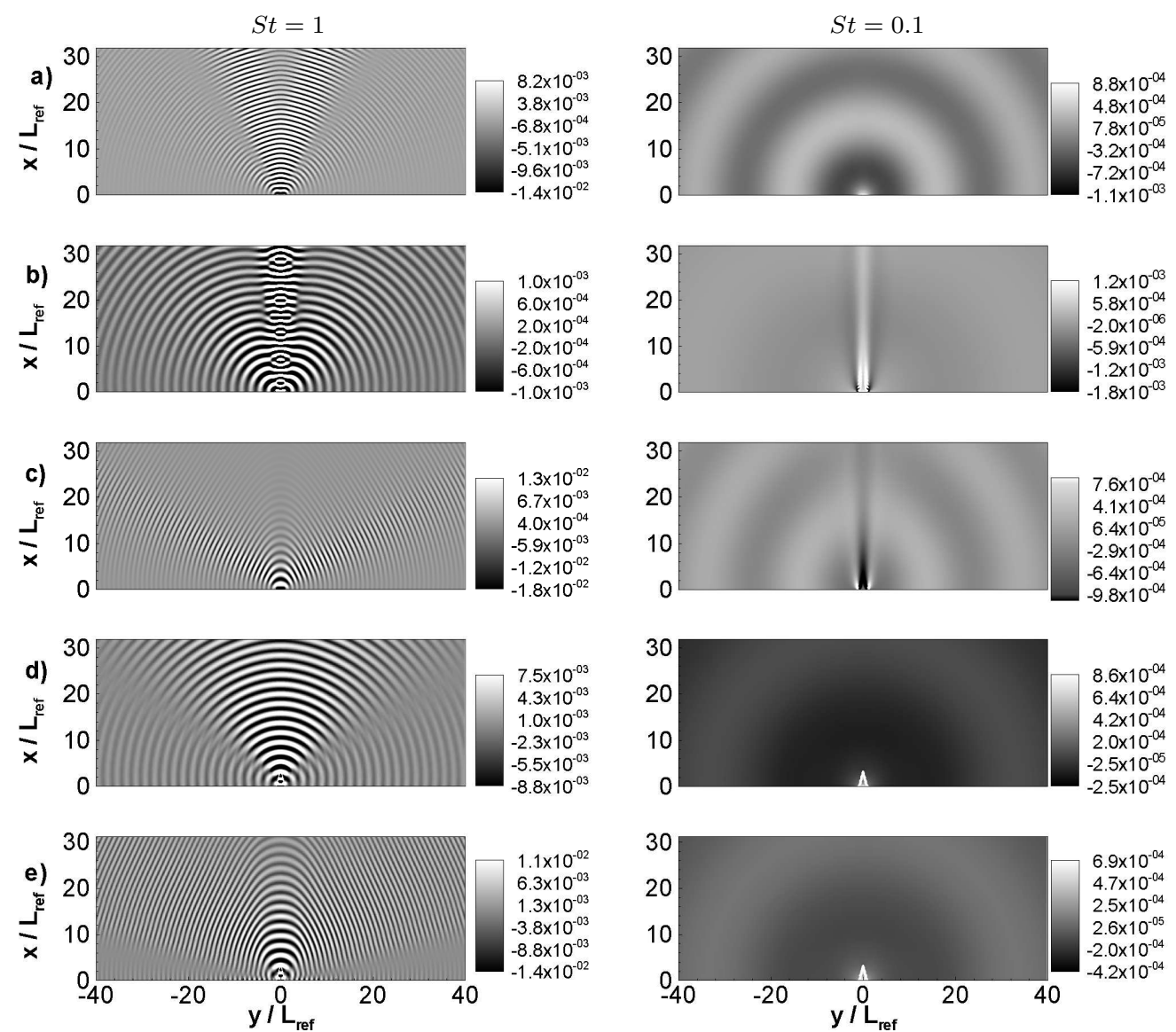

Fig. 7: Instantaneous dilatation field $L_{r e f} \boldsymbol{\nabla} \cdot \boldsymbol{u} / c_{u}$ for a) Case NR1, b) Case NR2, c) Case NR3, d) Case $\mathrm{R} 4 \mathrm{a}, \mathrm{e})$ Case R5 and $u_{i n} / c_{u}=0.04$. 


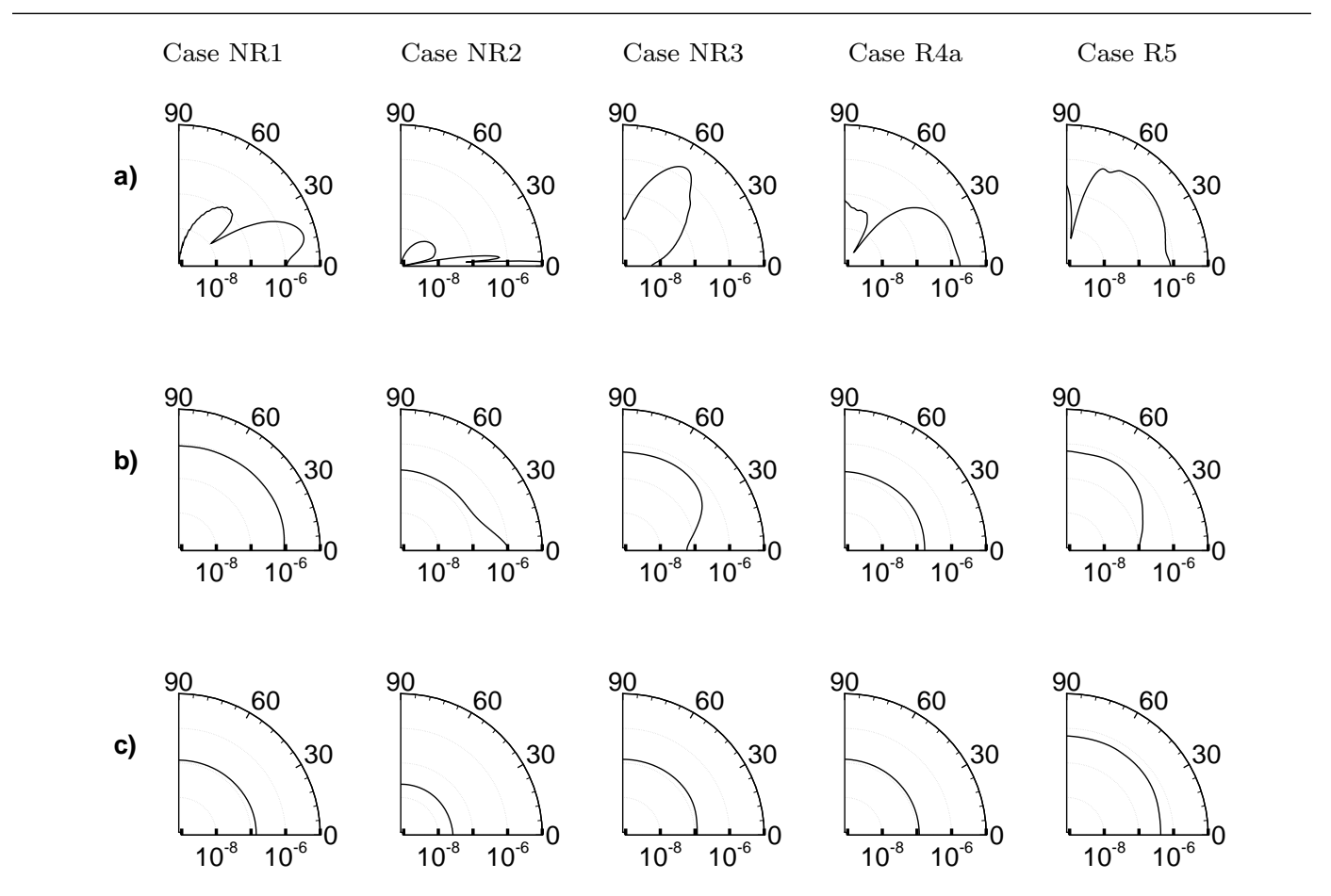

Fig. 8: Non-dimensional RMS pressure $p_{r m s}{ }^{2} / \rho_{r e f}{ }^{2} c_{u}{ }^{4}$ for a) $S t=1$, b) $S t=0.05$, c) $S t=0.02$ and $u_{i n} / c_{u}=0.04$ at $20 L_{r e f}$ radius. 

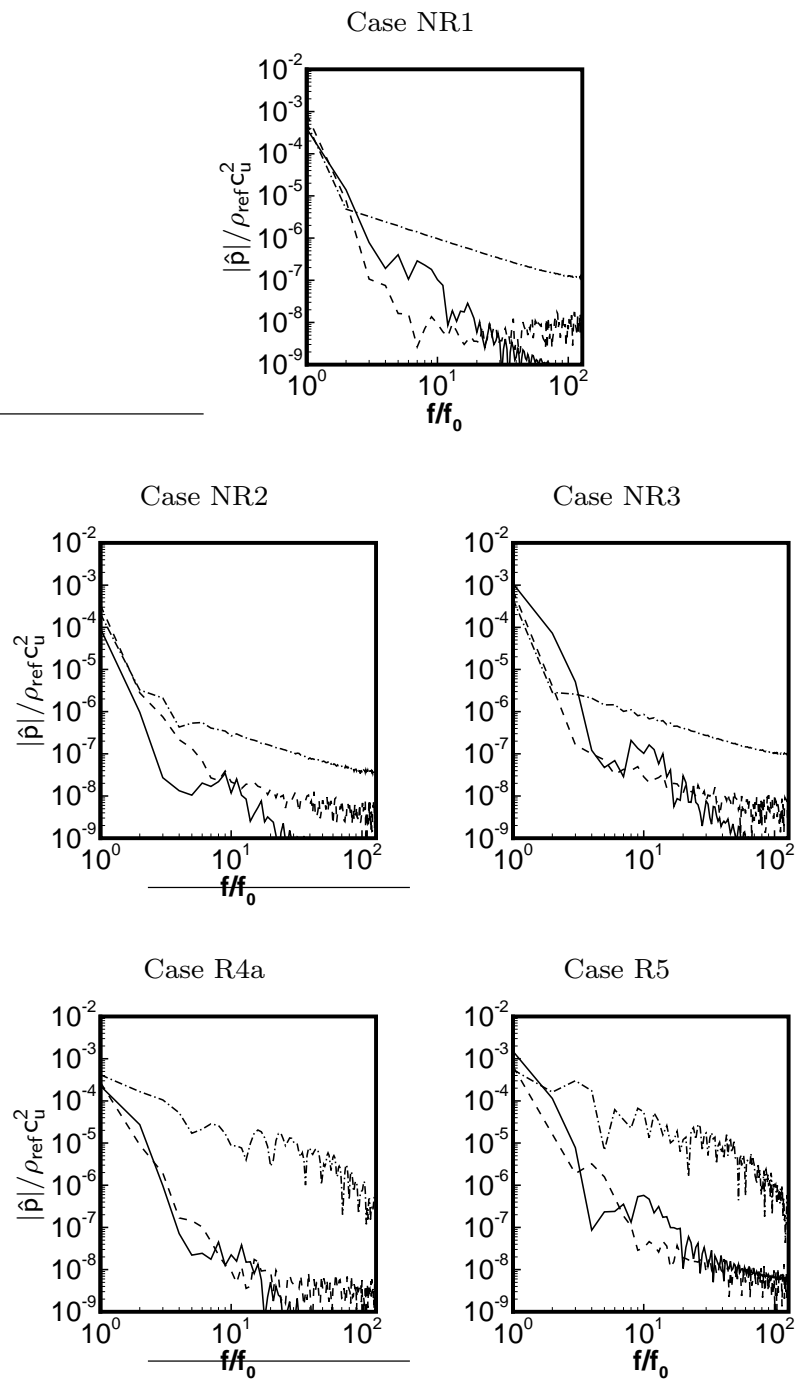

Fig. 9: Non-dimensional pressure amplitude at $x / L_{r e f}=16$ and $y / L_{r e f}=20$ versus non-dimensional frequency for $S t=1$ (solid line), $S t=0.1$ (dashed) and $S t=0.02$ (dash-dot). 
a)

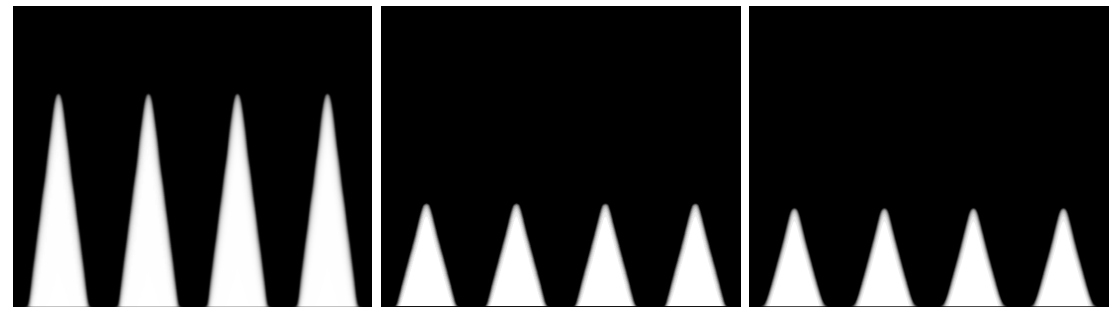

b)

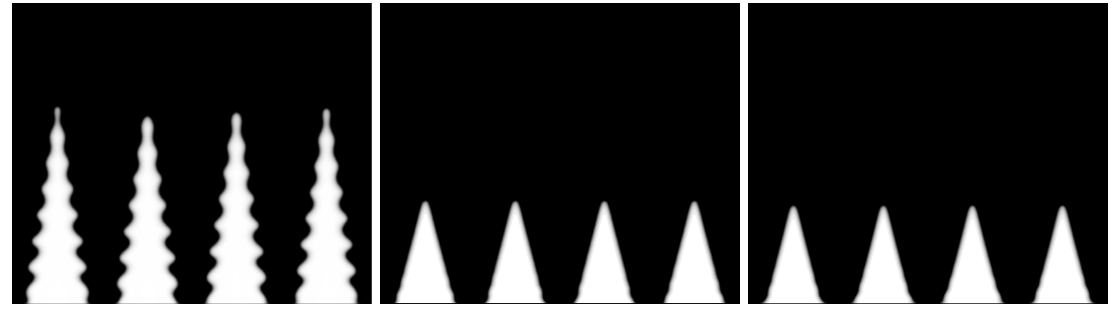

c)

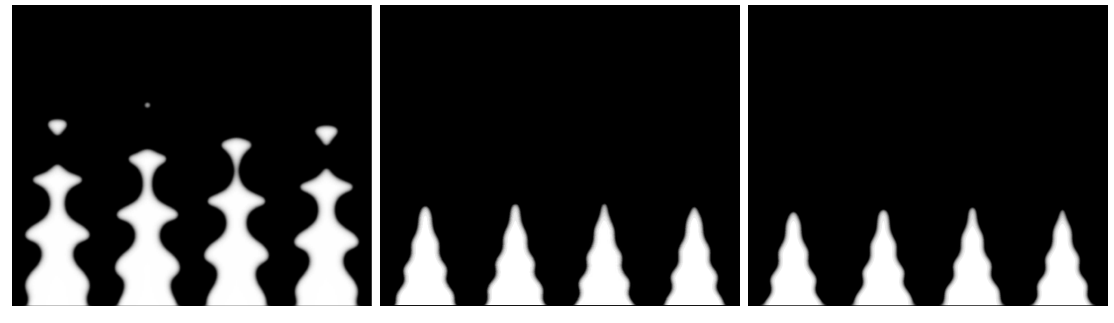

d)

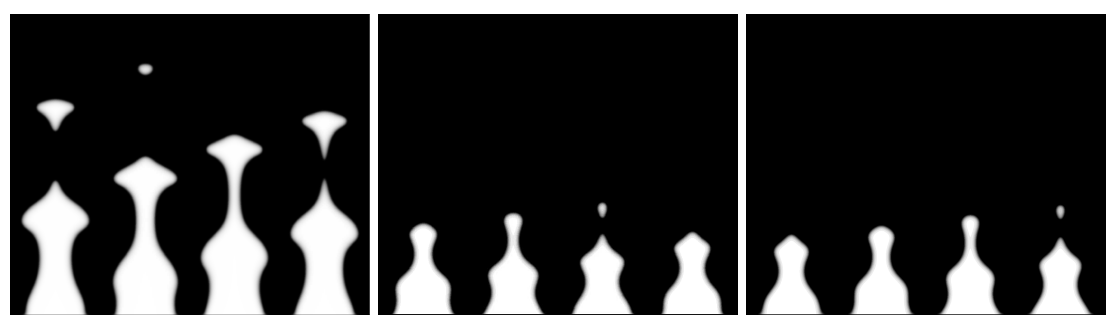

e)

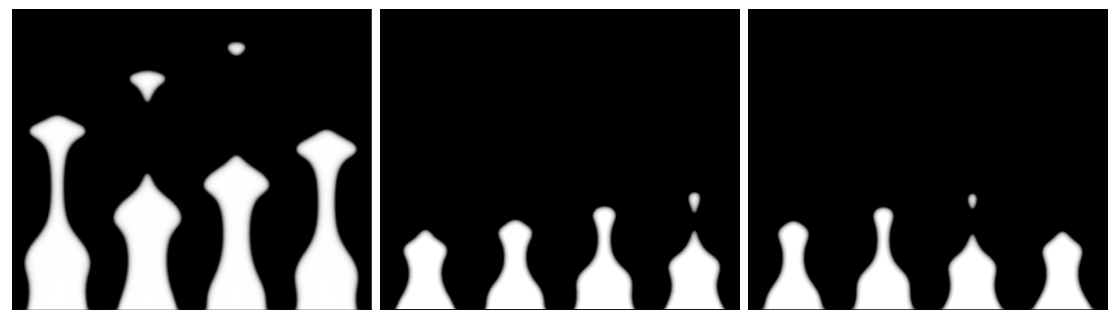

Fig. 10: Time evolution of unburnt mass fraction (black - burnt; white - unburnt), thresholded at $50 \%$ at $S t$ of a) 1 , b) 0.1 , c) 0.05 , d) 0.025 e) 0.02 . For each case, time evolves with a constant time interval from the leftmost frame to the rightmost frame. 

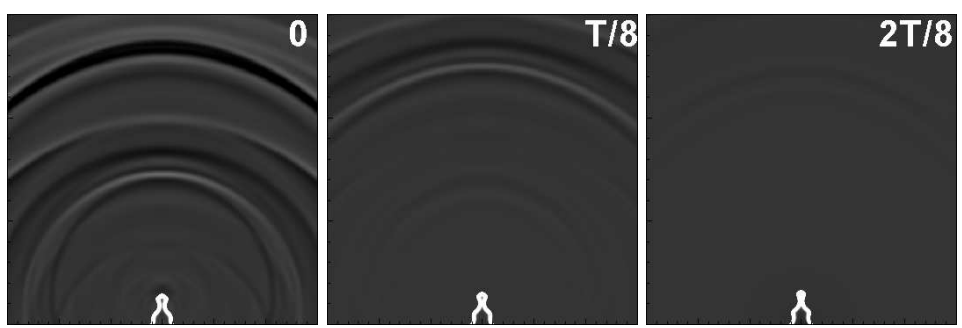

$3 T / 8$
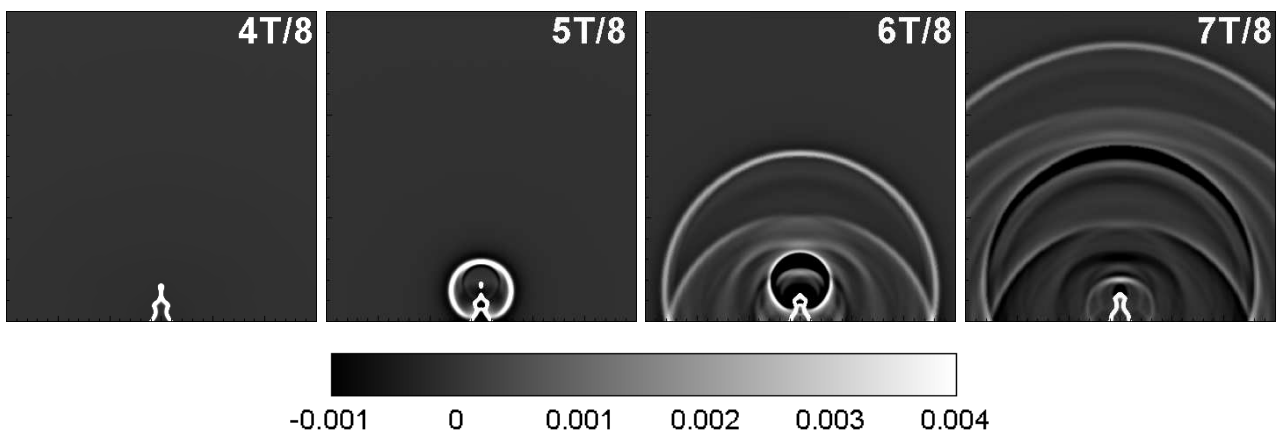

Fig. 11: Instantaneous dilatation field $L_{r e f} \nabla \cdot \boldsymbol{u} / c_{u}$ for Case R4a, $S t=0.02$ and $u_{i n} / c_{u}=0.04$. 


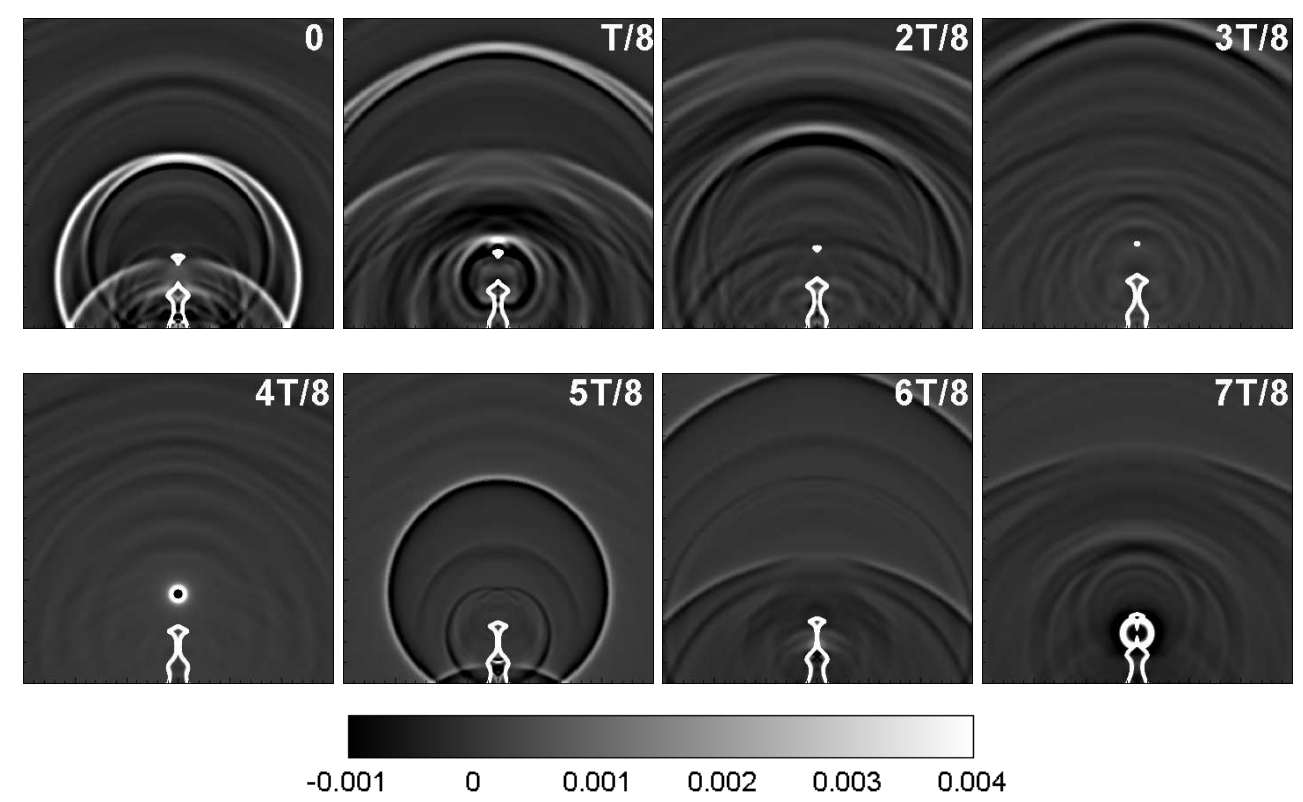

Fig. 12: Instantaneous dilatation field $L_{r e f} \nabla \cdot \boldsymbol{u} / c_{u}$ for Case R4b, St $=0.02$ and $u_{i n} / c_{u}=0.08$. 

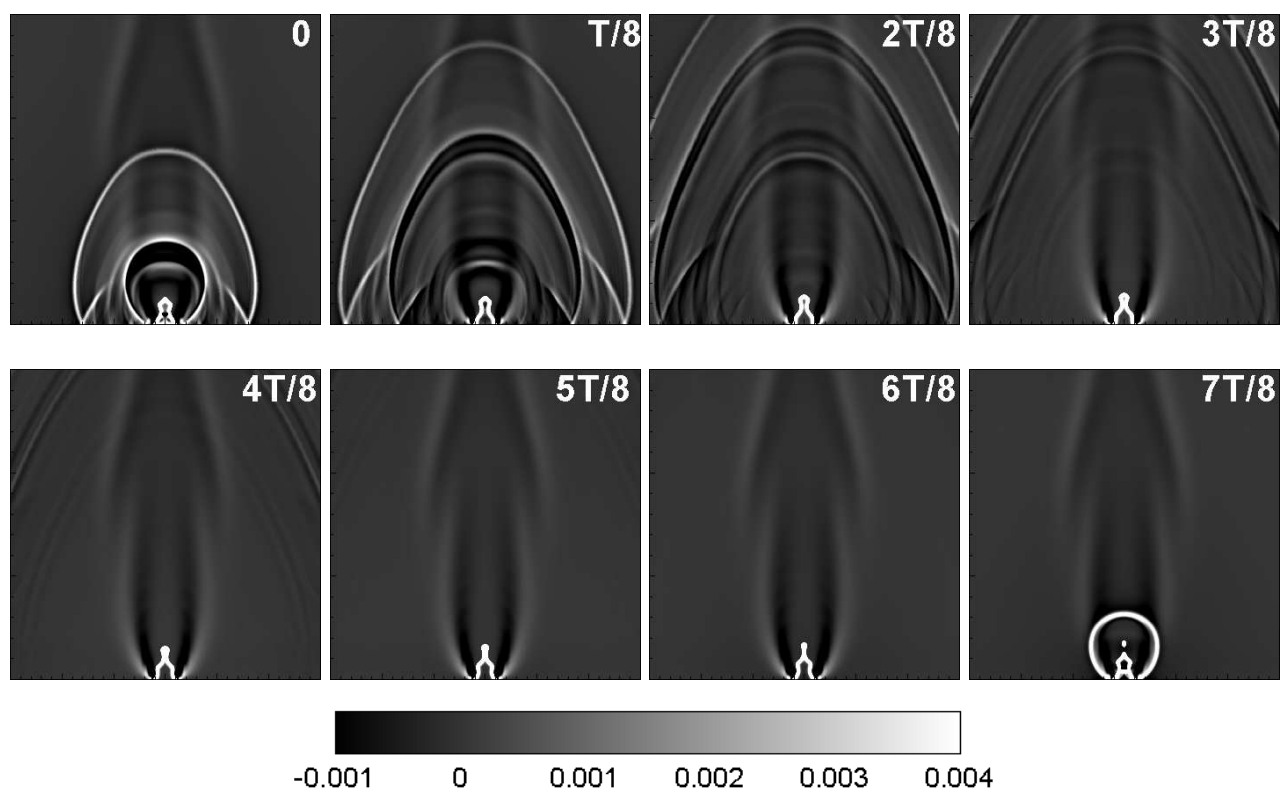

Fig. 13: Instantaneous dilatation field $L_{r e f} \boldsymbol{\nabla} \cdot \boldsymbol{u} / c_{u}$ for Case R5, St $=0.02$ and $u_{i n} / c_{u}=0.04$. 

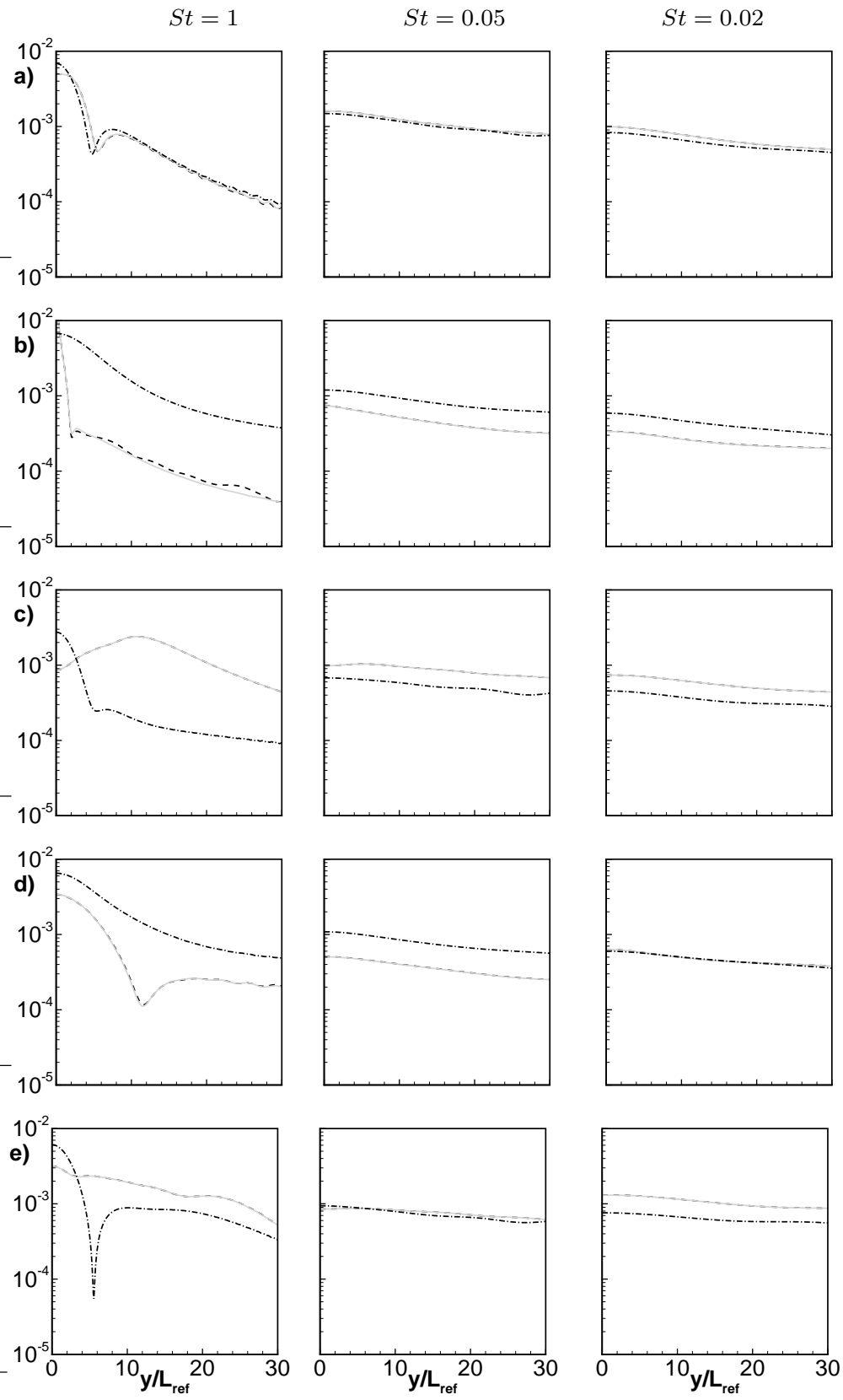

Fig. 14: Comparison of complete solution of Lighthill's equation for $|\hat{p}|$ at the forcing frequency (solid line), simulation results (dashed), solution of Lighthill's equation including only the inflow boundary term (dashdot) for a) Case NR1, b) Case NR2, c) Case NR3, d) Case R4a, e) Case R5 and $u_{i n} / c_{u}=0.04$ at $x / L_{r e f}=8$. Note that the simulation results is often obscured by the solution of Lighthill's equation. 

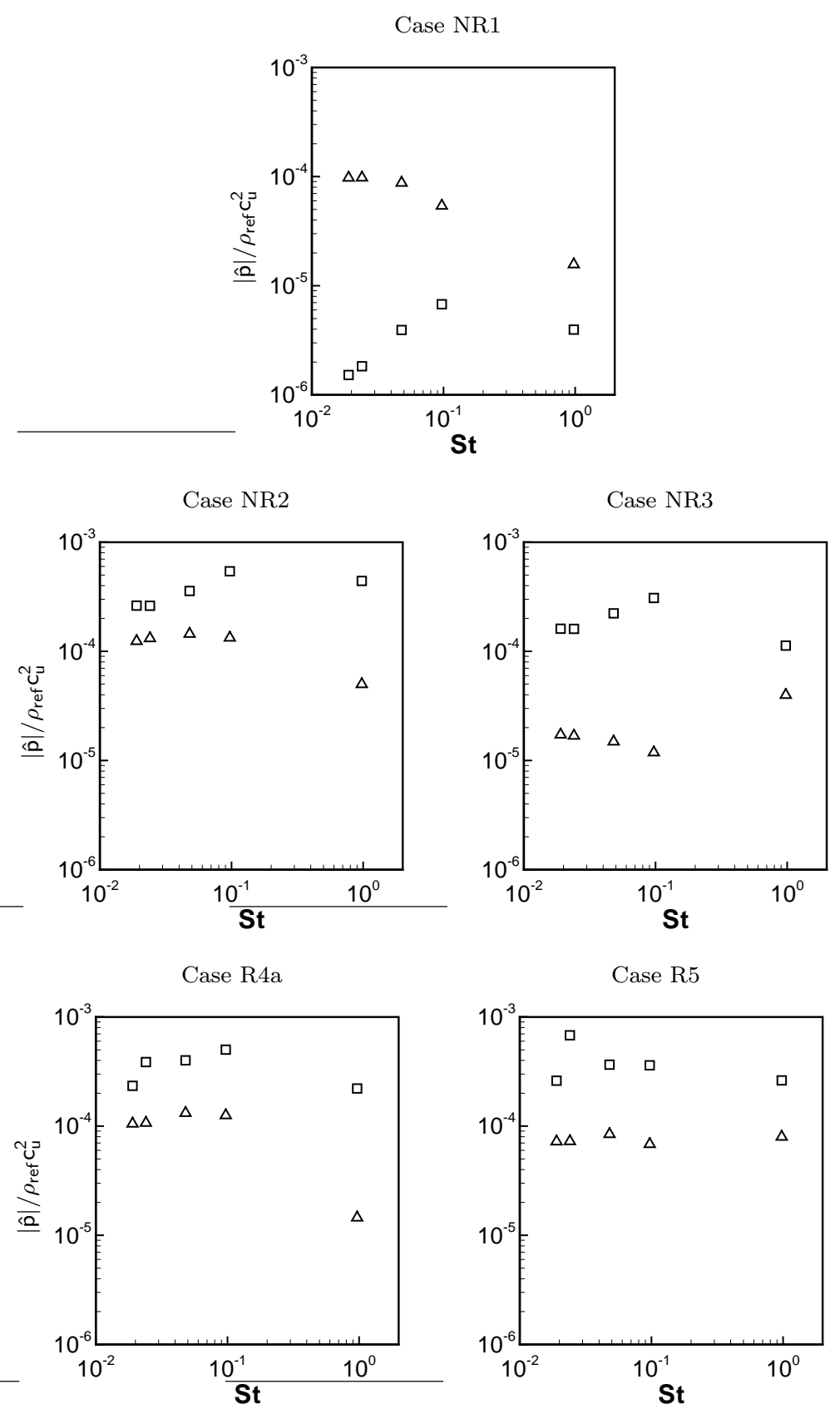

Fig. 15: Magnitude of $\hat{p}_{s t}(\Delta)$ and $\hat{p}_{e x}(\square)$ at the forcing frequency versus Strouhal number $S t$ at $(x=$ $\left.8 L_{r e f}, y=30 L_{r e f}\right), u_{i n} / c_{u}=0.04$. 

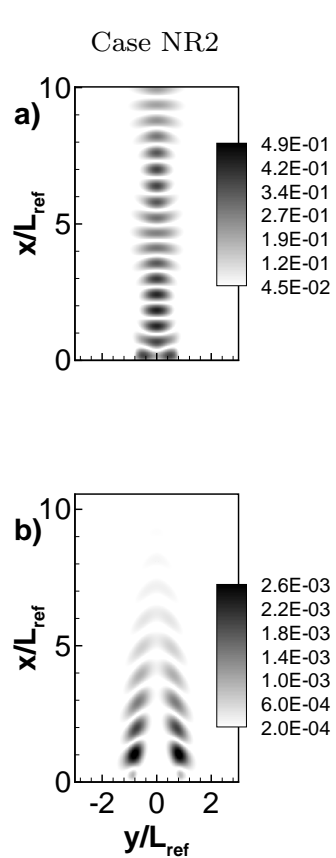

Case NR3

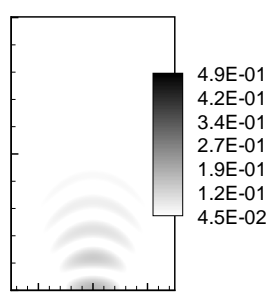

Case R4a
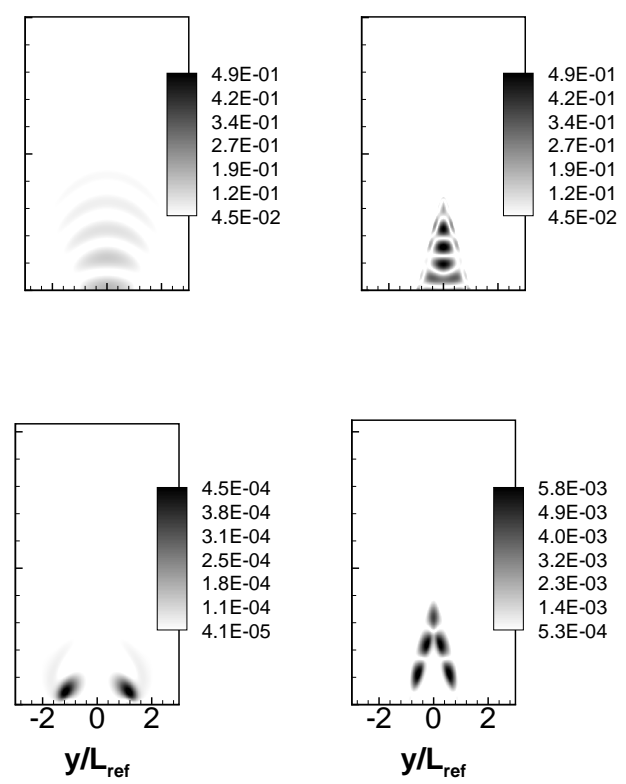
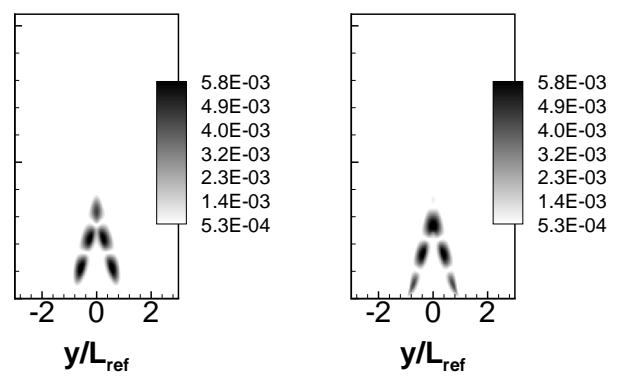

Fig. 16: Magnitude of the excess density term, at the forcing frequency in equation 18 for a) $S t=1$ and b) $S t=0.02$. 


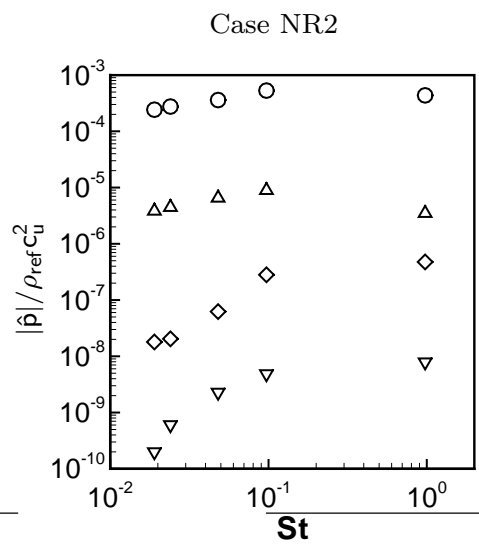

Case NR3

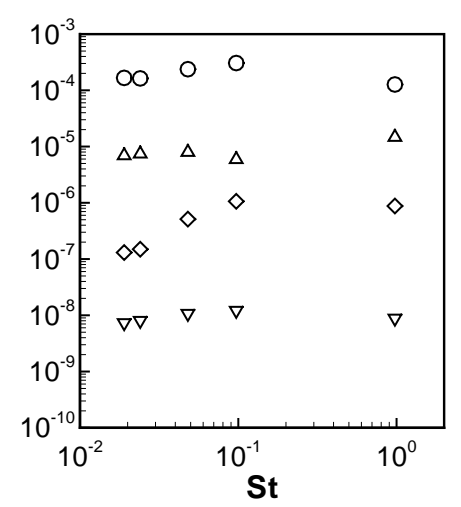

Fig. 17: Magnitude of $\hat{p}_{t h}(\Delta), \hat{p}_{v i s}(\nabla), \hat{p}_{\text {ex } 1}(\diamond)$ and $\hat{p}_{\text {ex } 2}(\circ)$ at $\left(x=8 L_{r e f}, y=30 L_{r e f}\right)$, at the forcing frequency versus Strouhal number $S t$ for non-reacting cases. 


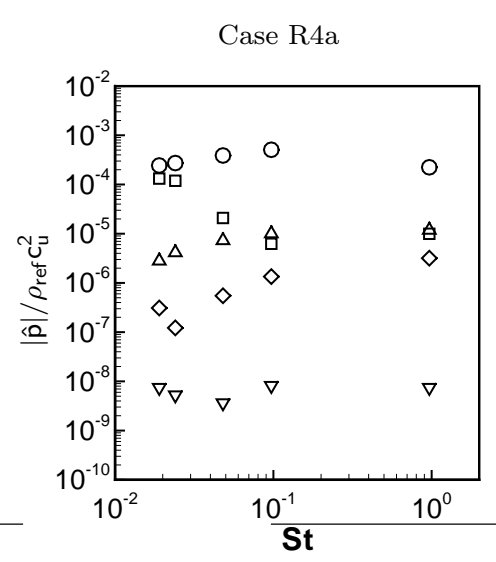

Case R5

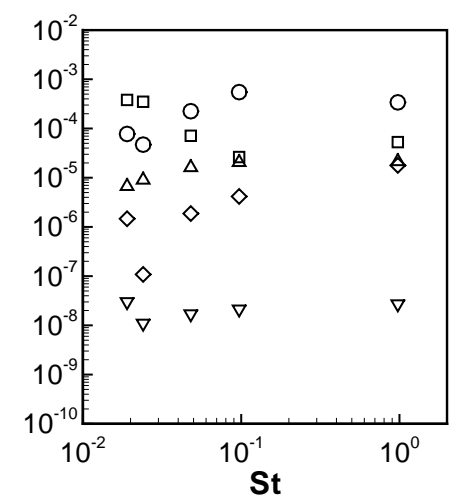

Case R4b

Case R4c
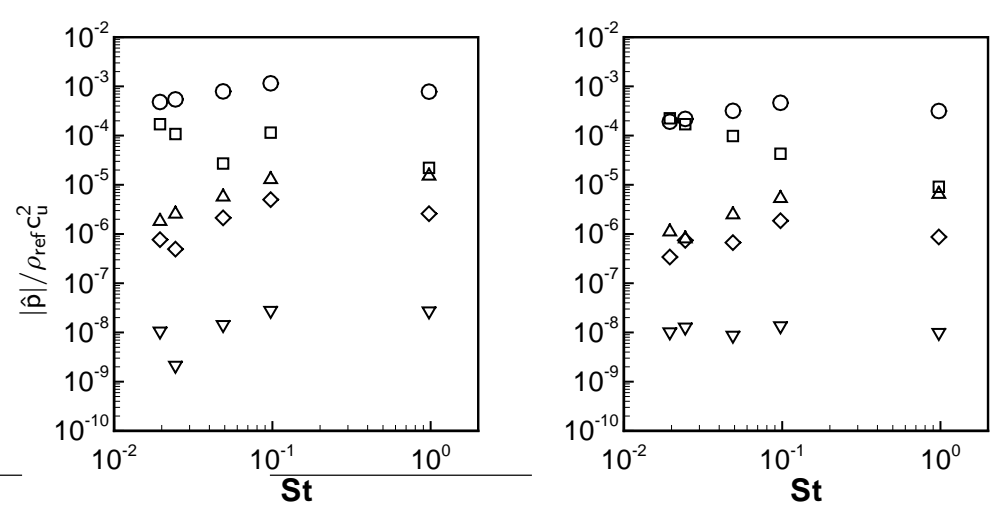

Case R4d

Case R4e
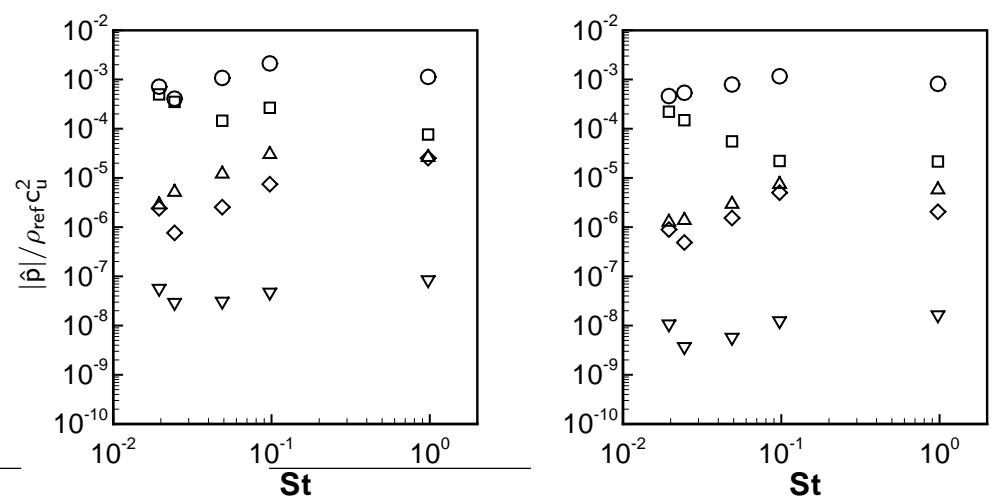

Fig. 18: Magnitude of $\hat{p}_{h r}(\square), \hat{p}_{t h}(\Delta), \hat{p}_{v i s}(\nabla), \hat{p}_{e x 1}(\diamond)$ and $\hat{p}_{e x 2}(\circ)$ at $\left(x=8 L_{r e f}, y=30 L_{r e f}\right)$, at the forcing frequency versus Strouhal number $S t$ for combusting cases. 
Acknowledgements The authors acknowledge the generous support of the Australian Research Council (ARC) and the European Centre for Research and Advanced Training in Scientific Computation (CERFACS, www.cerfacs.fr), in providing the authors with the source code for NTmix. Use of the facilities of the Victorian Partnership for Advanced Computing (VPAC) is also acknowledged.

\section{References}

1. Baum, M.: Etude de l'allumage et de la structure des flammes turbulentes. Ph.D. thesis, Ecole Centrale Paris (1994)

2. Bodony, D.J., Lele, S.K.: On using large-eddy simulation for the prediction of noise from cold and heated turbulent jets. Phys. Fluids 17 (2005)

3. Bourlioux, A., Cuenot, B., Poinsot, T.: Asymptotic and numerical study of the stabilization of diffusion flames by hot gas. Combust. Flame 120(1-2), 143-59 (2000)

4. Brear, M.J., Nicoud, F., Talei, M., Giauque, A., Hawkes, E.R.: Disturbance energy transport and sound production in gaseous combustion. J. Fluid Mech. 707, 53-73 (2012)

5. Bui, T.P., Schröder, W., Meinke, M.: Numerical analysis of the acoustic field of reacting flows via acoustic perturbation equations. Computers and Fluids 37(9), 1157-1169 (2008)

6. Candel, S.: Combustion dynamics and control: progress and challenges. Proc. Combust. Inst. 29(1), 1-28 (2002)

7. Candel, S., Durox, D., Ducruix, S., Birbaud, A.L., Noiray, N., Schuller, T.: Flame dynamics and combustion noise: progress and challenges. Int. J. Aeroacoustics 8(1\&2), 1-56 (2009)

8. Candel, S., Durox, D., Schuller, T.: Flame interactions as a source of noise and combustion instabilities. In: 10th AIAA/CEAS Aeroacoustics Conference, Paper number: 2004-2928, 2004-2928, pp. 1444-1454 (2004)

9. Chiu, H.H., Summerfield, M.: Theory of combustion noise. Acta Astronautica 1(7-8), 967-984 (1974)

10. Clavin, P., Siggia, E.D.: Turbulent premixed flames and sound generation. Combust. Sci. Technol. 78, 147-155 (1991)

11. Colonius, T., Lele, S.K.: Computational aeroacoustics: progress on nonlinear problems of sound generation. Prog. Aerosp. Sci. 40, 345-416 (2004)

12. Colonius, T., Lele, S.K., Moin, P.: Sound generation in a mixing layer. J. Fluid Mech. 330, 375-409 (1997)

13. Corjon, A., Poinsot, T.: A model to define aircraft separations due to wake vortex encounter. In: 13th AIAA Applied Aerodynamics Conference. AIAA paper 95-1776., pp. 117-124 (1995)

14. Corjon, A., Poinsot, T.: Behavior of wake vortices near ground. AIAA J. 35(5), 849-855 (1997)

15. Crighton, D.G., Dowling, A.P., Ffowcs Williams, J.E., Heckl, M., Leppington, F.G., Bartram, J.F.: Modern methods in analytical acoustics lecture notes (1992)

16. Cuenot, B., Bedet, B., Corjon, A.: NTMIX3D user's guide manual, Preliminary Version $1.0(1997)$

17. Doak, P.E.: Analysis of internally generated sound in continuous materials: 2. a critical review of the conceptual adequacy and physical scope of existing theories of aerodynamic noise, with special reference to supersonic jet noise 1. J. Sound Vib. 25(2), 263-335 (1972)

18. Dowling, A.P.: Modern Methods in Analytical Acoustics, chap. Thermoacoustic sources and instabilities, pp. 378-403. Springer (1992)

19. Dowling, A.P., Stow, S.R.: Acoustic analysis of gas turbine combustors. J. Propul. Power 19(5), 751-764 (2003)

20. Duffy, D.G.: Green's Functions with Applications. Chapman \& Hall/CRC (2001) 
21. Ffowcs Williams, J.E., Hawking, D.L.: Sound generated by turbulence and surfaces in arbitrary motion. Phil. Trans. R. Soc. A264, 321-342 (1969)

22. Freund, J.B.: Noise sources in a low-Reynolds-number turbulent jet at Mach 0.9. J. Fluid Mech. 438, 277-305 (2001)

23. Goldstein, M.E.: Aeroacoustics of turbulent shear flows. Ann. Rev. Fluid Mech. 16, 263 285 (1984)

24. Goldstein, M.E.: A generalized acoustic analogy. J. Fluid Mech. 488, 315-333 (2003)

25. Hassan, H.A.: Scaling of combustion-generated noise. J. Fluid Mech. 66(3), 445-453 (1974)

26. Hirsch, C., Wäsle, J., Winkler, A., Sattelmayer, T.: A spectral model for the sound pressure from turbulent premixed combustion. Proc. Combust. Inst. 31(1), 1435-1441 (2007)

27. Howe, M.S.: Acoustics of fluid-structure interactions. Cambridge University Press (1998)

28. Hurle, I.R., Price, R.B., Sugden, T.M., Thomas, A.: Sound emission from open turbulent premixed flames. Proc. Roy. Soc. 303(1475), 409-427 (1968)

29. Ihme, M., Pitsch, H.: On the generation of direct combustion noise in turbulent nonpremixed flames. Int. J. Aeroacoustics 11(1), 25-78 (2012)

30. Ihme, M., Pitsch, H., Bodony, D.J.: Radiation of noise in turbulent non-premixed flames. Proc. Combust. Inst. 32(1), 1545-1553 (2009)

31. Jiang, X., Avital, E.J., Luo, K.H.: Direct computation and aeroacoustic modelling of a subsonic axisymmetric jet. J. Sound Vib. 270, 528-538 (2004)

32. Karimi, N., Brear, M.J., Jin, S.H., Monty, J.P.: Linear and non-linear forced response of a conical, ducted, laminar premixed flame. Combust. Flame 156(11), 2201-2212 (2009)

33. Kidin, N., Librovich, V., Macquisten, M., Roberts, J., Vuillermoz, M.: Possible acoustic source in turbulent combustion. Dynamics of Reactive Systems. Part 1: Flames pp. 336348 (1988)

34. Kidin, N., Librovich, V., Roberts, J., Vuillermoz, M.: On sound sources in turbulent combustion. Dynamics of Flames and Reactive Systems pp. 343-355 (1984)

35. Lieuwen, T.: Modeling premixed combustion-acoustic wave interactions: A review. J. Propul. Power 19(5), 765-781 (2003)

36. Lieuwen, T., Yang, V. (eds.): Combustion Instabilities in Gas Turbine Engines: Operational Experience, Fundamental Mechanisms, And Modeling. Prog. Astronaut. Aeronaut., AIAA (2006)

37. Lighthill, M.J.: On sound generation aerodynamically I. General theory. Proc. Roy. Soc. 211, 564-587 (1951)

38. Lilley, G.M.: On the noise from air jets. AGARD CP 131 (1971)

39. Lodato, G., Domingo, P., Vervisch, L.: Three-dimensional boundary conditions for direct and large-eddy simulation of compressible viscous flows. J. Comput. Phys. 227(10), 51055143 (2008)

40. Moore, P., Slot, H., Boersma, B.J.: Investigation of the behavior of noise sources in heated jets. Advances in Turbulence XI pp. 395-397 (2007)

41. Morfey, C.L.: Amplification of aerodynamic noise by convected flow inhomogeneities. J. Sound Vib. 31(4), 391-397 (1973)

42. Morfey, C.L., Wright, M.C.M.: Extensions of Lighthill's acoustic analogy with application to computational aeroacoustics. Proc. Roy Soc. A-Math Phy. 463(2085), 2101-2127 (2007)

43. Myers, M.K.: Transport of energy by disturbances in arbitrary steady flows. J. Fluid Mech. 226, 383-400 (1991)

44. Phillips, O.M.: On the generation of sound by supersonic turbulent shear layers. J. Fluid Mech. 9, 1-25 (1960)

45. Poinsot, T., Veynante, D.: Theoretical and numerical combustion, Second Edition. RT Edwards, Inc. (2005) 
46. Poinsot, T.J., Lele, S.K.: Boundary conditions for direct simulations of compressible viscous flows. J. Comput. Phys. 101(1), 104-129 (1992)

47. Schuller, T., Durox, D., Candel, S.: Dynamics of and noise radiated by a perturbed impinging premixed jet flame. Combust. Flame 128(1-2), 88-110 (2002)

48. Schuller, T., Durox, D., Candel, S.: Self-induced combustion oscillations of laminar premixed flames stabilized on annular burners. Combust. Flame 135(4), 525-537 (2003)

49. Schwarz, A., Janicka, J. (eds.): Combustion Noise. Springer (2009)

50. Smith, M.J.T.: Aircraft noise. Cambridge University Press (2004)

51. Strahle, W.C.: On combustion generated noise. J. Fluid Mech. 49(2), 399-414 (1971)

52. Strahle, W.C.: Some results in combustion generated noise. J. Sound Vib. 23(1), 113-125 (1972)

53. Strahle, W.C.: Combustion noise. Prog. Energy Combust. 4, 157-176 (1978)

54. Talei, M., Brear, M.J., Hawkes, E.R.: Sound generation by laminar premixed flame annihilation. J. Fluid Mech. 679, 194-218 (2011)

55. Talei, M., Brear, M.J., Hawkes, E.R.: A parametric study of sound generation by laminar premixed flame annihilation. Combust. Flame, 159(2), 757-769 (2012)

56. Talei, M., Hawkes, E.R., Brear, M.J.: A direct numerical simulation study of frequency and Lewis number effects on sound generation by two-dimensional forced laminar premixed flames. Proc. Combust. Inst. p. http://dx.doi.org/10.1016/j.proci.2012.07.034 (2012)

57. Torregrosa, A.J., Broatch, A., Martín, J., Monelletta, L.: Combustion noise level assessment in direct injection diesel engines by means of in-cylinder pressure components. Meas. Sci. Technol. 18, 2131-2142 (2007)

58. Yoo, C., Wang, Y., Trouvé, A., Im, H.: Characteristic boundary conditions for direct simulations of turbulent counterflow flames. Combustion Theory and Modelling 9(4), 617-646 (2005)

59. Yoo, C.S., Im, H.G.: Characteristic boundary conditions for simulations of compressible reacting flows with multi-dimensional, viscous and reaction effects. Combust. Theor. Model. 11(2), 259-286 (2007)

60. Zhao, W., Frankel, S.H.: Numerical simulations of sound radiated from an axisymmetric premixed reacting jet. Phyis. Fluids 13, 2671-2681 (2001) 


\section{University Library}

\section{- M M N E R VA A gateway to Melbourne's research publications}

Minerva Access is the Institutional Repository of The University of Melbourne

Author/s:

Talei, M;Brear, MJ;Hawkes, ER

Title:

A comparative study of sound generation by laminar, combusting and non-combusting jet flows

Date:

2014-08-01

\section{Citation:}

Talei, M., Brear, M. J. \& Hawkes, E. R. (2014). A comparative study of sound generation by laminar, combusting and non-combusting jet flows. THEORETICAL AND COMPUTATIONAL FLUID DYNAMICS, 28 (4), pp.385-408. https://doi.org/10.1007/s00162-014-0324-7.

Persistent Link:

http://hdl.handle.net/11343/283321 